\title{
Colored HOMFLY polynomials for the pretzel knots and links
}

\author{
A. Mironov, ${ }^{a, b, c, d}$ A. Morozov ${ }^{b, c, d}$ and A. Sleptsov ${ }^{b, c, d, e}$ \\ a Theory Department, Lebedev Physics Institute, \\ Leninsky prospekt, Moscow 119991, Russia \\ ${ }^{b}$ ITEP, \\ Bol. Cheremushkinskaya, Moscow 117218, Russia \\ ${ }^{c}$ National Research Nuclear University MEPhI, \\ Kashirskoe sh., Moscow 115409, Russia \\ ${ }^{d}$ Institute for Information Transmission Problems, \\ Bol. Karetny, Moscow 127994, Russia \\ ${ }^{e}$ Laboratory of Quantum Topology, Chelyabinsk State University, \\ Chelyabinsk 454001, Russia \\ E-mail: mironov@lpi.ru, morozov@itep.ru, sleptsov@itep.ru
}

\begin{abstract}
With the help of the evolution method we calculate all HOMFLY polynomials in all symmetric representations $[r]$ for a huge family of (generalized) pretzel links, which are made from $g+1$ two strand braids, parallel or antiparallel, and depend on $g+1$ integer numbers. We demonstrate that they possess a pronounced new structure: are decomposed into a sum of a product of $g+1$ elementary polynomials, which are obtained from the evolution eigenvalues by rotation with the help of rescaled $S U_{q}(N)$ Racah matrix, for which we provide an explicit expression. The generalized pretzel family contains many mutants, undistinguishable by symmetric HOMFLY polynomials, hence, the extension of our results to non-symmetric representations $R$ is a challenging open problem. To this end, a non-trivial generalization of the suggested formula can be conjectured for entire family with arbitrary $g$ and $R$.
\end{abstract}

Keywords: Quantum Groups, Chern-Simons Theories, Topological Field Theories

ARXIV EPRINT: 1412.8432 


\section{Contents}

1 Introduction 1

2 Warm-up examples 4

2.1 Genus $g=1$, fundamental representation, two parallel strands 4

2.2 Genus $g=1$, fundamental representation, antiparallel strands 5

2.3 HOMFLY in the fundamental representation at arbitrary genus 6

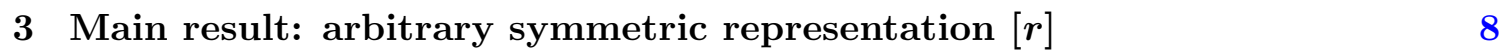

4 Comments on the main result (3.1) 11

5 Matrices $a_{k m}$ and $\bar{a}_{k m}$ as universal Racah matrix 14

6 Checks of the main conjecture (3.1) 17

$\begin{array}{lll}6.1 & \text { Jones polynomials } & 17\end{array}$

$\begin{array}{ll}\text { 6.2 Comparing with families of knots/links } & 17\end{array}$

6.3 Comparing with arbitrary pretzels at concrete representations 18

$\begin{array}{ll}6.4 \text { Reduction and expansion tests } & 18\end{array}$

6.5 Table: Rolfsen and Thistlethwaite vs. pretzel [64] 19

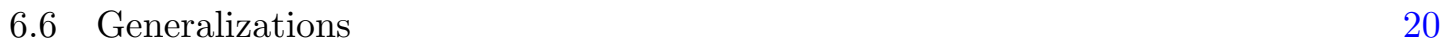

$\begin{array}{llr}7 & \text { Conclusion } & 20\end{array}$

A Symmetrically colored HOMFLY for generalized pretzel knots 22

B List of coefficients $a_{k m}, \bar{a}_{k m}$ and $\overline{\bar{a}}_{k m} \quad 26$

\section{Introduction}

Despite impressive progress during the last years [1-11, 13-18], evaluation of colored HOMFLY polynomials [19-26] for particular knots and links remains a non-trivial exercise. It makes use of a variety of advanced methods of modern theoretical physics, however, they still remain not powerful enough for this task, which in turn helps to further develop these methods. Knot polynomials are interesting, because they are the simplest possible example of Wilson-loop averages in gauge (Chern-Simons) theory [27] on one hand and are close relatives of the holomorphic conformal blocks on the other hand. They depend on variety of parameters, and the purpose is to study and understand these dependencies, which are already known to satisfy various interesting equations, generalizing the previously known ones in simpler (quantum) field theories, [28]. 


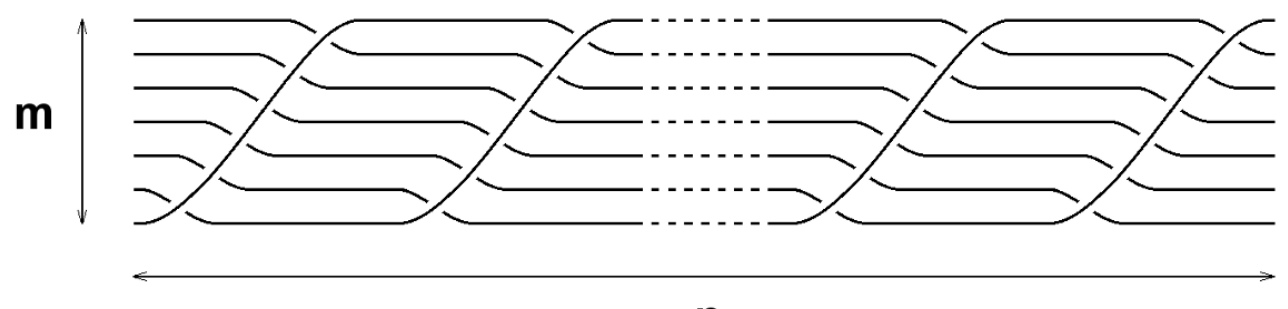

$\mathbf{n}$

Figure 1. Torus $[\mathrm{m}, \mathrm{n}]$ braid.

Especially interesting are results obtained for entire families of knots or links. The most famous example is the two-parametric set of torus knots and links, formed by a nonintersecting lines, wrapping around a torus respectively $m$ and $n$ times along its two noncontractible cycles. The link diagrams for a torus knot/link is just a closure of especially simple $m$-strand braid, figure 1 .

In this case, the HOMFLY polynomial in arbitrary representation $R$ is given by the Rosso-Jones formula [29, 30],

$$
H_{R}^{[m, n]}(q, A)=q^{2 m n \varkappa_{R}} A^{(m-1) n|R|} \cdot \sum_{Q \in R^{\otimes m}} C_{R Q} \lambda_{Q}^{2 n / m} \chi_{Q}^{*}(q, A)
$$

Here the sum goes over Young diagrams $Q$ of the size $|Q|=m|R|$, the quantum dimensions of the corresponding representations of the linear group $G L(N)$ are the values of Schur functions at the "topological locus" in the space of time-variables, $\chi_{Q}^{*}=\chi_{Q}\left\{p_{k}=\frac{\left\{A^{k}\right\}}{\left\{q^{k}\right\}}\right\}$ where $A=q^{N}$ and $\{x\}=x-x^{-1}$, so that the quantum number is $[x]=\frac{\left\{q^{x}\right\}}{\{q\}}$ and the "DGR differential" [31] is $D_{i}=\frac{\left\{A q^{i}\right\}}{\{q\}}$. Parameters $\lambda_{Q}$ are associated eigenvalues of the quantum $\mathcal{R}$-matrix, made from the eigenvalues $\varkappa_{Q}$ of the cut-and-join operator [32, 33]

$$
\lambda_{Q} \sim q^{\varkappa_{Q}}, \quad \varkappa_{Q}=\sum_{(i, j) \in Q}(j-i)
$$

where there is an arbitrary factor in $\lambda_{Q}$ that depends on the framing. Finally, the coefficients $C_{R Q}$ are defined from the expansion of the Adams transform of characters $\chi_{R}$ :

$$
\left(\chi_{R}\left\{p_{m k / l}\right\}\right)^{l}=\sum_{Q \in R^{\otimes m}} C_{R Q} \chi_{Q}\left\{p_{k}\right\}
$$

where $l=$ maximal common divisor $(m, n)$ is the number of components in the torus link. For coprime $n$ and $m$ one has a knot and $l=1$.

When $A=q^{N}$, eq. (1.1) can be also recast in an $N$-fold integral [34, 35]

$$
H_{R}^{[m, n]}(q, A) \sim \int \prod_{i=1}^{N} d \mu_{i} \exp \left\{\frac{\mu_{i}^{2}}{2 m n \hbar}\right\} \prod_{i<j}^{N} \sinh \left(\frac{\mu_{i}-\mu_{j}}{2 m}\right) \sinh \left(\frac{\mu_{i}-\mu_{j}}{2 n}\right) \chi_{R}\left[\operatorname{diag}\left(e^{\mu_{i}}\right)\right]
$$

where the symmetry between $m$ and $n$ is explicitly restored. 


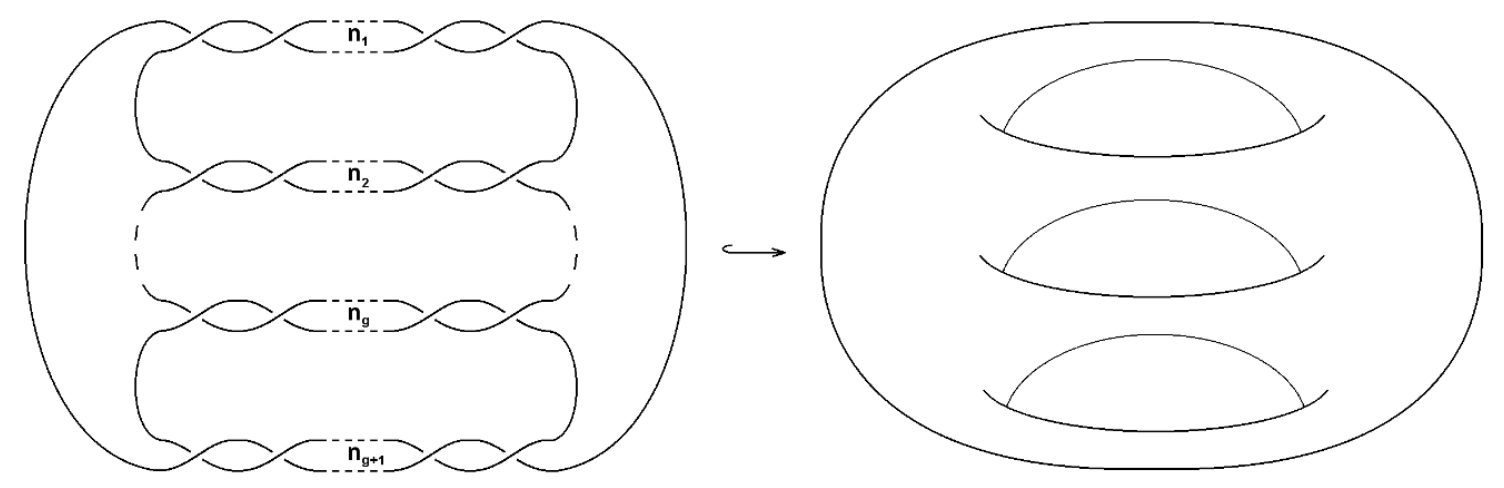

Figure 2. Pretzel link or knot of genus $g=3$.

Eq. (1.1) can be directly generalized to superpolynomials [36-38], depending on one extra parameter $t$, with the Schur functions promoted to the Macdonald polynomials, though associated deformation of the matrix model (1.4) is still unavailable.

Also a mystery remains what makes the torus links so special: despite numerous attempts no comparably explicit formulas for all representations $R$ at once were yet found for any other family. What was done, however, the simple dependence on $n$ (but not on $m$ ) in (1.1) was interpreted in [37] as an evolution in the length of an $m$-strand braid, and this fact remains true for any such braid inside any, arbitrarily complicated knot or link [39]: dependence on its length $n$ will enter only through a linear combination of $\lambda_{Q}^{2 n / m}$. Still the coefficients $C_{R Q} \chi_{Q}^{*}$ can be quite sophisticated. To define them, one needs "initial conditions" for the evolution, i.e. explicit knowledge of knot polynomials for a few particular values of $n$. Despite an extreme naiveness of the evolution method it allowed one to study certain interesting families, in particular, the important family of twist knots [39] and led to a discovery of a very important "differential structure" [40, 41] of arbitrary knot polynomials, which seems related to the original ideas in [31], and led to a number of impressive advances in knot calculus, at least, for symmetric representations [42-51]. (However, attempts to generalize the matrix model $(1.4)$ in $[52,53]$ and to describe nonsymmetric representations in [54-56] are still only partly successful.)

The goal of the present paper is to extend previous calculations to a much richer family, which, taken as a total, looks like a straightforward generalization of the torus knots, and thus provides more chances to guess the relevant way to generalize (1.1) and, perhaps, even (1.4). These are knots and links formed by wrapping around a surface of genus $g$ without self-intersections, which can be different from $g=1$. The simplest set of this type has a link diagram (see figure 2), consisting of $g+1$ two-strand braids, and thus has $g+1$ different evolution parameters $n_{1}, \ldots, n_{g+1}$ (for $g=1$ everything depends on the sum $n=n_{1}+n_{2}$ ). In literature (see [57]) this family is known as the pretzel knots and links. The family is actually split into subfamilies, differing by mutual orientation of strands in the braids. For certain orientations the family has a cyclic symmetry $n_{k} \longrightarrow n_{k+1}$. In fact, if one considers only symmetric representations, the symmetry is actually enhanced to arbitrary permutations of $n_{k}$, links/knots related by these permutations are actually mutants [58] and symmetric HOMFLY polynomials are the same for them. 


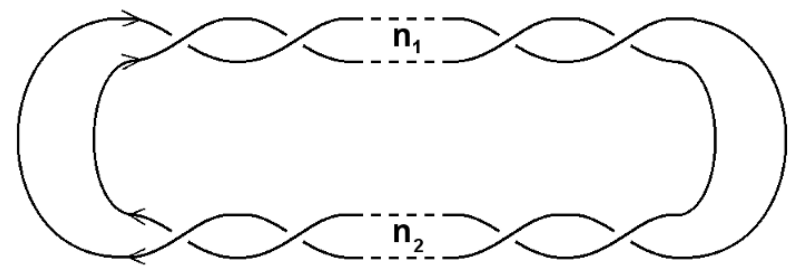

Figure 3. Two parallel strands: torus links and knots.

Notations. For the sake of convenience, we repeat here our notations once again:

$$
\begin{array}{rlrl}
\chi_{Q}^{*} & =\chi_{Q}\left\{p_{k}=\frac{\left\{A^{k}\right\}}{\left\{q^{k}\right\}}\right\} & \\
\{x\} & =x-x^{-1}, & {[x]} & =\frac{\left\{q^{x}\right\}}{\{q\}}, \quad D_{i}=\frac{\left\{A q^{i}\right\}}{\{q\}} \\
\lambda_{Q} & \sim \epsilon_{Q} q^{\varkappa_{Q}}, & \varkappa_{Q} & =\sum_{(i, j) \in Q}(i-j)
\end{array}
$$

where $\epsilon_{Q}$ is a sign factor, which will be fixed latter (it is always +1 in the Rosso-Jones case). As soon as throughout the text only the Schur functions at the topological locus, $\chi_{Q}^{*}$ are used (for the only exception see the third paragraph of section 4), from now on, we omit the asterisk and use just the notation $\chi_{Q}$.

\section{Warm-up examples}

\subsection{Genus $g=1$, fundamental representation, two parallel strands}

We begin with this simplest example, which is the simplest possible case of the Rosso-Jones formula (1.1). In our family we should restrict it to two strands, $m=2$, so that

$$
H_{R}^{\left(n_{1}, n_{2}\right)}=\sum_{Q \vdash 2|R|} \lambda_{Q}^{n_{1}+n_{2}} \chi_{Q}
$$

and, in the fundamental representation,

$$
H_{[1]}^{\left(n_{1}, n_{2}\right)}=\lambda_{[2]}^{n_{1}+n_{2}} \chi_{[2]}+\lambda_{[11]}^{n_{1}+n_{2}} \chi_{[11]}
$$

with $\lambda_{[2]}=q / A$ and $\lambda_{[11]}=-1 /(q A)$ in the topological framing, figure 3 .

However, if one did not know the answer and looks at the problem from the point of view of the evolution method, it is necessary to consider the following anzatz:

$$
H_{[1]}^{\left(n_{1}, n_{2}\right)}=c_{11} \lambda_{[2]}^{n_{1}+n_{2}}+c_{10} \lambda_{[2]}^{n_{1}} \lambda_{[11]}^{n_{2}}+c_{01} \lambda_{[2]}^{n_{2}} \lambda_{[11]}^{n_{1}}+c_{00} \lambda_{[11]}^{n_{1}+n_{2}}
$$

with four unknown coefficients. Apparent symmetry between $n_{1}$ and $n_{2}$ implies that $c_{10}=$ $c_{01}$, and looking at the picture one understands that the answer depends only on $n_{1}+n_{2}$, thus actually $c_{10}=c_{01}=0$. The two remaining parameters can be found from the two 
initial conditions: for $n_{1}+n_{2}= \pm 1$ one gets the unknot, with the HOMFLY polynomial equal to $\chi_{[1]}$, i.e.

$$
c_{11} \lambda_{[2]}^{ \pm 1}+c_{00} \lambda_{[11]}^{ \pm 1}=\chi_{[1]}
$$

and

$$
\begin{aligned}
& c_{11}=\frac{\frac{1}{\lambda_{[11]}}-\lambda_{[11]}}{\frac{\lambda_{[2]}}{\lambda_{[11]}}-\frac{\lambda_{[11]}}{\lambda_{[2]}}} \cdot \chi_{[1]}=\frac{\{A q\}}{\left\{q^{2}\right\}} \cdot \chi_{[1]}=\chi_{[2]} \\
& c_{00}=\frac{\lambda_{[2]}-\frac{1}{\lambda_{[2]}}}{\frac{\lambda_{[2]}}{\lambda_{[11]}}-\frac{\lambda_{[11]}}{\lambda_{[2]}}} \cdot \chi_{[1]}=\frac{\{A / q\}}{\left\{q^{2}\right\}} \cdot \chi_{[1]}=\chi_{[11]}
\end{aligned}
$$

what brings us back to

$$
H_{[1]}^{\left(n_{1}, n_{2}\right)}=\lambda_{[2]}^{n_{0}+n_{1}} \chi_{[2]}+\lambda_{[11]}^{n_{0}+n_{1}} \chi_{[11]}
$$

(one can easily check the third obvious initial condition: for $n_{0}+n_{1}=0$ one gets a pair of disconnected unknots with the HOMFLY polynomial $\left.\chi_{[1]}^{2}=\chi_{[2]}+\chi_{[11]}\right)$. Since $\chi_{[2]}+\chi_{[11]}=\frac{\{A q\}+\{A / q\}}{\left\{q^{2}\right\}} \cdot \chi_{[1]}=\chi_{[1]}^{2}$ (the relation is actually valid beyond the topological locus), one can rewrite this in an identical, but more sophisticated form:

$$
\begin{aligned}
H_{[1]}^{\left(n_{1}, n_{2}\right)}= & \frac{\chi_{[2]}^{2}+\chi_{[2]} \chi_{[11]}}{\chi_{[1]}^{2}} \cdot \lambda_{[2]}^{n_{1}+n_{2}}+\frac{\chi_{[2]} \chi_{[11]}-\chi_{[2]} \chi_{[11]}}{\chi_{[1]}^{2}}\left(\lambda_{[2]}^{n_{1}} \lambda_{[11]}^{n_{2}}+\lambda_{[2]}^{n_{2}} \cdot \lambda_{[11]}^{n_{1}}\right) \\
& +\frac{\chi_{[11]}^{2}+\chi_{[2]} \chi_{[11]}}{\chi_{[1]}^{2}} \lambda_{[11]}^{n_{1}+n_{2}}= \\
= & \sum_{i=0}^{2} C_{[1]}^{i} \cdot\left(\lambda_{[2]}^{n_{1}+n_{i}} \lambda_{[11]}^{n_{i+1}+n_{2}}+\text { permutations of } n_{1}, n_{2}\right)
\end{aligned}
$$

where

$$
C_{[1]}^{i}=\frac{1}{\chi_{[1]}^{2}}\left(\chi_{[2]}^{2-i} \chi_{[11]}^{i}+(-)^{i} \chi_{[2]} \chi_{[11]}\right)
$$

and the only permutations from the two different groups of indices are included.

\subsection{Genus $g=1$, fundamental representation, antiparallel strands}

Before going to higher $g$ and higher representations, we consider the same genus-one twostrand example, but now with antiparallel strands, figure 4.

This configuration is possible only if $n_{1}+n_{2}$ is even, and it is always a link, hence generically the corresponding HOMFLY polynomials depend on two representations, $R_{1} \otimes$ $R_{2}$. The two parallel strands, considered in the previous section, correspond to $R_{2}=R_{1}=$ [1], while for the antiparallel strands the fundamental HOMFLY implies that $R_{2}$ is rather conjugate of $R_{1}, R_{2}=\overline{R_{1}}=\overline{[1]}=\left[q^{N-1}\right]$. This is still a particular case of the Rosso-Jones formula (1.1), since it is valid at any representation.

From the point of view of the evolution method, one has now $[1] \otimes[1]=$ Adjoint+singlet, and according to [39] the two relevant eigenvalues are $\lambda_{0}=1$ and $\lambda_{a d j}=-A$. As the initial condition one can take the pair of unknots at $n_{1}+n_{2}=0$ and the Hopf link at $n_{1}+n_{2}=2$. 


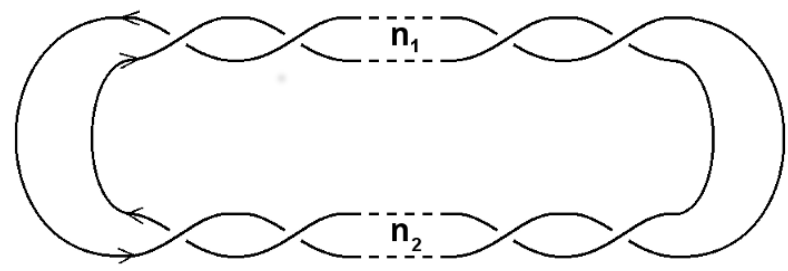

Figure 4. Two antiparallel strands: torus links.

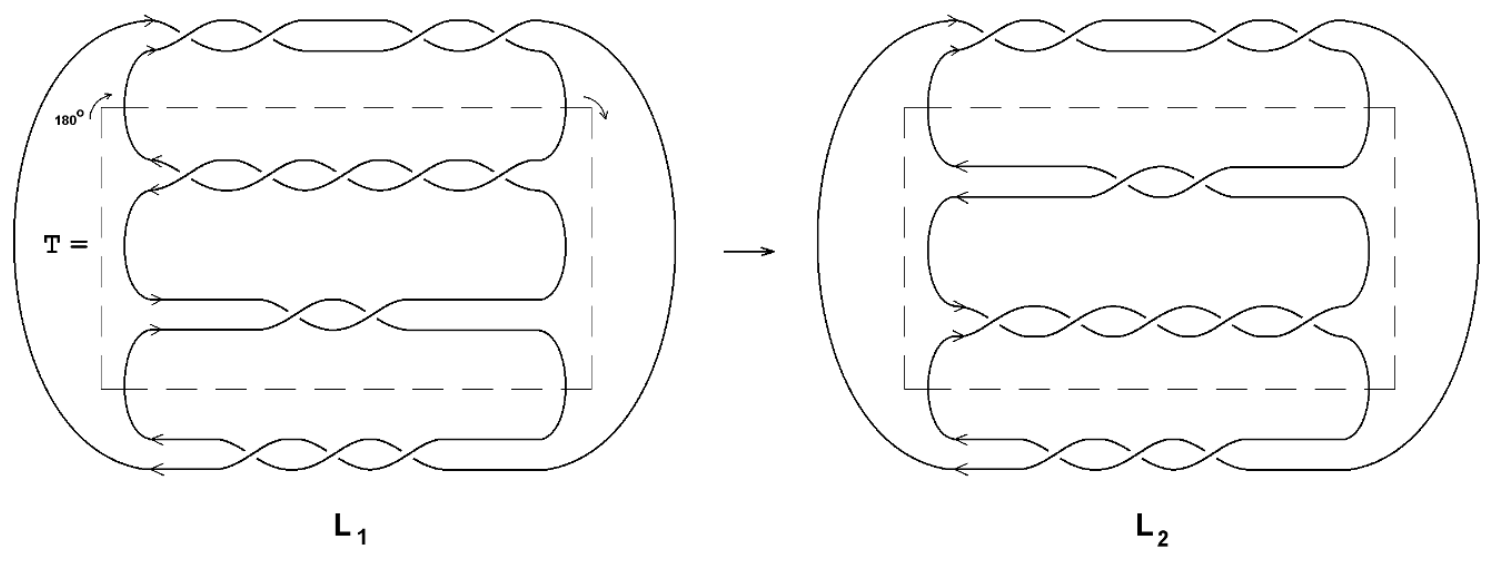

Figure 5. Mutant knots.

\subsection{HOMFLY in the fundamental representation at arbitrary genus}

Now we can switch to arbitrary genus. Again there will be different options to choose orientations of particular strands. While for links the freedom is rather big, for knots the orientation depends only on the genus. For odd $g$ one can make all the braids parallel, while for even $g$ exactly one should be antiparallel. Moreover, the corresponding parameter, which we choose to be $n_{g / 2+1}$, should be even. We also consider the case when all the braids are antiparallel.

The next question is what happens to the symmetry $n_{1} \leftrightarrow n_{2}$. For $g>1$ the polynomials depend on all $n_{i}$ independently, and there is only a cyclic symmetry when all $n_{k} \longrightarrow n_{k+1}$. However, as we shall see, the answer in the fundamental representation is actually symmetric in all $n_{k}$. In fact this should not come as a surprise, because permutation of the two adjacent $n_{k}$ 's is just a knot mutation. Indeed, (by definition following [59]) let we have oriented link $L_{1}$ which contains a marked tangle $T$ (see figure 5 ). Remove $T$, rotate it by $180^{\circ}$ about the axe transversal to the plane of the picture and glue it back in position to form a new link $L_{2}$. If $L_{1} \neq L_{2}$ then they are called mutants of each other, and this operation is called mutation. Since the HOMFLY polynomials in symmetric representations do not distinguish the mutant knots [58], with the help of mutation one can permute $n_{k} \leftrightarrow n_{k+1}$. This enhanced symmetry reduces the number of necessary initial conditions and, thus, more formulas can be obtained and more are the chances to observe regularities, leading to discovery of generic expressions. Many are provided, by putting one of parameters, say $n_{g}$, equal to zero, then the knot/link reduces to a composite one, which 
enjoys the decomposition property

$$
\frac{H_{R}^{\text {composite }}}{\chi_{R}}=\prod_{\text {components }} \frac{H_{R}^{\text {component }}}{\chi_{R}}
$$

To these patterns, one can add already known particular examples, like twist knots.

All this makes explicit calculation by the evolution method possible at genera $g=$ $1,2,3,4$, at least in the fundamental representation. And this is enough to discover the structure and obtain the general formulas for the HOMFLY polynomial in the fundamental representation:

$$
H_{[1]}^{\left(n_{1}, \ldots, n_{g+1}\right)}=\sum_{i=0}^{g+1} C_{[1]}^{i} \cdot\left(\lambda_{0}^{n_{1}+\ldots+n_{i}} \lambda_{1}^{n_{i+1}+\cdots+n_{g+1}}+\text { permutations of } n_{i}\right)
$$

where again only permutations from the two different groups of indices are included and the coefficients $C_{[1]}^{i}$ are:

- odd $g$, all braids parallel:

$$
\begin{aligned}
C_{[1]}^{i} & =\frac{1}{\chi_{[1]}^{g+1}}\left(\chi_{[2]}^{i} \chi_{[11]}^{g+1-i}+(-)^{i} \chi_{[2]} \chi_{[11]} z^{g-1}\right) \\
\lambda_{0} & =\lambda_{[11]}, \quad \lambda_{1}=\lambda_{[2]}
\end{aligned}
$$

- even $g$, all braids antiparallel:

$$
\begin{aligned}
C_{[1]}^{\bar{i}} & =\frac{1}{z^{i} \chi_{[1]}^{g+1}}\left(\chi_{[2]}^{i} \chi_{[11]}+(-)^{i} \chi_{[2]} \chi_{[11]}^{i}\right) \\
\lambda_{0} & =1, \lambda_{1}=\lambda_{a d j}
\end{aligned}
$$

- even $g$, all braids parallel, except for one antiparallel, $n_{g / 2+1}$ should be even; in this case each term in the sum, (2.10) is a product of $g$ factors $\lambda_{[11]}^{n_{i}}$ and $\lambda_{[2]}^{n_{j}}$ and one factor either $\lambda_{0}^{n_{k}}=1$ or $\lambda_{a d j}^{n_{k}}$, in these two different cases the coefficients $C_{[1]}^{i}$ being

$$
\begin{aligned}
\text { e.v. } \lambda_{0}=1: \quad C_{[1]}^{i} & =\frac{1}{\chi_{[1]}^{g+1}}\left(\chi_{[2]}^{i} \chi_{[11]}^{g-i}+(-)^{i} \chi_{[2]} \chi_{[11]} z^{g-2}\right) \\
\text { e.v. } \lambda_{a d j}=A: \quad C_{[1]}^{i} & =\frac{\chi_{[2]} \chi_{[11]}}{z^{2} \chi_{[1]}^{g+1}}\left(\chi_{[2]}^{i} \chi_{[11]}^{g-i}+(-)^{i+1} z^{g}\right) \\
& =\frac{\{q\}^{g-1}\{A q\}\{A / q\}}{\{A\}^{g+1}}\left(\chi_{[2]}^{i} \chi_{[11]}^{g-i}+(-)^{i+1} z^{g}\right) .
\end{aligned}
$$

Here $z=\frac{1}{[2]} \chi_{[1]}$.

The common factor $\{A q\}\{A / q\}$ in the second formula in (2.15) is required by the differential expansion.

The structure of these formulas is very simple: there is the "main contribution", the first terms in each line, which is in a clear one-to-one correspondence with the combination 
of $\lambda$-factors, plus "corrections" which look a little less universal. In fact, the same structure survives in higher representations, at least symmetric.

Formulas (2.12)-(2.15) provide an exhaustive description of the fundamental HOMFLY for all the pretzel knots.

For $N=2$ there is no orientation dependence (except for a simple framing factor), ${ }^{1}$ and all the four formulas turn into one:

$$
C_{[1]}^{(i)} \stackrel{A=q^{2}}{=} \frac{[3]}{[2]^{2 g+2}}\left([3]^{i-1}+(-)^{i}\right)
$$

what gives $1,0,[3], \frac{[3][4]}{[2]}=[5]+1, \ldots$ for $i=0,1,2,3, \ldots$ respectively. (Note that at $N=2$ we have $z=1, \chi_{[2]}=[3]$ and $\chi_{[11]}=1$.) Eq. (2.16) is in perfect accordance with the result in $[60,61]$, as well as with those in [62].

\section{Main result: arbitrary symmetric representation $[r]$}

Our main result is an explicit combinatorial formula for unreduced HOMFLY of arbitrary pretzel link in symmetric representation. The formula includes only three ingredients and looks like

$$
H_{R}^{n_{1}, \ldots, n_{g+1}}=\sum_{X} \operatorname{dim}_{q} X \prod_{i=1}^{g+1} \sum_{Y} \mathcal{A}_{X Y} \lambda_{Y}^{n_{i}}
$$

Now we define the ingredients.

- Eigenvalues. Since we construct the pretzel link with the help of 2-strand braids only, there are only two possible orientations for such a braid: parallel and

antiparallel. The parallel strands correspond to the product of two symmetric representations $[r]$ :

$$
[r] \otimes[r]=\oplus_{m=0}^{r}[r+m, r-m]
$$

The corresponding evolution eigenvalues $\lambda$ in the topological framing are equal to

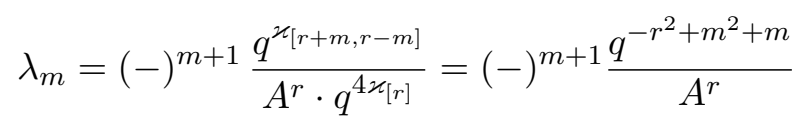

Similarly, the antiparallel strands correspond to the product of symmetric representation and its conjugate:

$$
[r] \otimes \overline{[r]}=\oplus_{m=0}^{r}\left[2 m, m^{N-2}\right]
$$

and the corresponding evolution eigenvalues $\bar{\lambda}$ in the topological framing are equal to

$$
\bar{\lambda}_{m}=\left(-q^{m-1} A\right)^{m}
$$

\footnotetext{
${ }^{1}$ This is because the orientation independence is due to a group theory argument: for $\mathrm{SU}(2)$ group the representation coincides with its conjugate. However, this is only the vertical framing that respects the group theory structures, and in the topological framing there is slight orientation dependence, that is, additional factors.
} 
- Dimensions. The quantum dimensions $\Delta_{m}$ of representations arising in (3.2) are equal to

$$
\Delta_{m}=\chi_{[r+m, r-m]}=\frac{[2 m+1]}{[r+m+1] ![r-m] !} \prod_{i=0}^{2 r-1} D_{j} \prod_{j=0}^{r-m-1} \frac{D_{j-1}}{D_{r+m+j}},
$$

while the quantum dimensions $\bar{\Delta}_{m}$ of representations arising in (3.4) are

$$
\bar{\Delta}_{m}=D_{2 m-1} \cdot\left(\prod_{j=0}^{m-2} \frac{D_{j}}{[j+2]}\right)^{2} \cdot D_{-1}
$$

- Universal matrix. The third constituent is a universal matrix $\mathcal{A}$ (we typically use the notation $a_{i j}$ for its matrix elements) which ultimately turns out to be related with the matrix of the quantum Racah coefficients (or, up to a factor, of the $6 j$-symbols). In fact, in order to describe all the pretzel links and knots we will need three different universal matrices $\mathcal{A}$. After some tedious calculations we have found the following explicit formulas for $\mathcal{A}$ :

$$
\begin{aligned}
& a_{k m}=\alpha_{k m} \cdot \mathcal{G} \\
& \bar{a}_{k m}=\alpha_{k m} \cdot \overline{\mathcal{G}} \\
& \overline{\bar{a}}_{k m}=\frac{\bar{\Delta}_{m}}{\Delta_{k}} a_{m k}
\end{aligned}
$$

where $\alpha_{k m}$ are the coefficients in the $S U_{q}(2)$ case (i.e. $A=q^{2}$ ), which does not differ between the parallel and antiparallel orientations:

$$
\begin{aligned}
\alpha_{k m}= & (-1)^{r+k+m}[2 m+1] \cdot \frac{([k] ![m] !)^{2}[r-k] ![r-m] !}{[r+k+1] ![r+m+1] !} \times \\
& \times \sum_{j=\max (r+m, r+k)}^{\min (r+k+m, 2 r)} \frac{(-1)^{j}[j+1] !}{[2 r-j] !([j-r-k] ![j-r-m] ![r+k+m-j] !)^{2}}
\end{aligned}
$$

and we introduce the following special functions

$$
\begin{aligned}
\mathcal{G} & =\frac{G(r-m) G(j+1)}{G(r+k+1) G(j-r-m)} \\
\overline{\mathcal{G}} & =\frac{D_{2 m-1}}{[2 m+1]} \cdot \frac{G(m)^{2} G(j+1)}{G(r+k+1) G(r+m+1) G(r+k+m-j)} \\
G(n) & =\frac{1}{[n] !} \prod_{i=-1}^{n-2} D_{i}=\frac{(A / q ; q)_{n}}{(q ; q)_{n}}
\end{aligned}
$$

where we used the symmetric q-Pochhammer symbol $(A ; q)_{n}=\prod_{j=0}^{n-1}\left\{A q^{j}\right\}$. At $A=q^{N}$, $G(n)$ becomes the q-binomial $\left(\begin{array}{c}N+n-2 \\ n\end{array}\right)_{q}$. 
Let us note that $\mathcal{G}$ and $\overline{\mathcal{G}}$ are equal to 1 when $A=q^{2}$, thus reducing (3.8) and (3.9) to (3.11). Particular examples of these matrices are given in appendix B.

Matrix (3.8) satisfies the weighted orthogonality relation

$$
\sum_{k=0}^{r} \bar{\Delta}_{k} \cdot a_{k m} a_{k m^{\prime}}=\Delta_{m} \delta_{m, m^{\prime}} .
$$

The dual relation is

$$
\sum_{m=0}^{r} \frac{a_{k m} a_{k^{\prime} m}}{\Delta_{m}}=\frac{\delta_{k, k^{\prime}}}{\bar{\Delta}_{k}} .
$$

Matrix (3.9) also satisfies the orthogonality conditions:

$$
\begin{aligned}
\sum_{k=0}^{r} \bar{\Delta}_{k} \cdot \bar{a}_{k m} \bar{a}_{k m^{\prime}} & =\bar{\Delta}_{m} \cdot \delta_{m, m^{\prime}}, \\
\sum_{m=0}^{r} \frac{\bar{a}_{k m} \bar{a}_{k^{\prime} m}}{\bar{\Delta}_{m}} & =\frac{\delta_{k, k^{\prime}}}{\bar{\Delta}_{k}}
\end{aligned}
$$

and matrix (3.10) satisfies the orthogonality conditions:

$$
\begin{gathered}
\sum_{k=0}^{r} \Delta_{k} \cdot \overline{\bar{a}}_{k m} \overline{\bar{a}}_{k m^{\prime}}=\bar{\Delta}_{m} \delta_{m, m^{\prime}} \\
\sum_{m=0}^{r} \frac{\overline{\bar{a}}_{k m} \overline{\bar{a}}_{k^{\prime} m}}{\bar{\Delta}_{m}}=\frac{\delta_{k, k^{\prime}}}{\Delta_{k}}
\end{gathered}
$$

The 0th rows of matrices (3.8) and (3.9) are equal to the quantum dimensions of the corresponding representations (3.2) and (3.4):

$$
\begin{aligned}
& a_{0 m}=\Delta_{m} \\
& \bar{a}_{0 m}=\bar{\Delta}_{m}
\end{aligned}
$$

Now we specify formula (3.1) for three possible cases of pretzel knots/links.

Antiparallel odd case. Let us consider the case when all parameters $n_{1}, \ldots, n_{g}$ are odd and all strand into constituent braids are antiparallel. This case is stand-alone and does not mix with any others, i.e. it is impossible to represent knot or link with $n_{1}, \ldots, n_{i}$ odd antiparallel 2-strand braids and $n_{i}, \ldots, n_{g}$ odd parallel or even (anti)parallel 2-strand braids. Since for all qualities standing for the antiparallel case we use "bar", we denote parameters in this case as $\overline{n_{1}}, \ldots, \overline{n_{g}}$. Concerning topological classification of this case we can point out the following: if the genus $g$ is odd then the result is a 2-component link, if the genus $g$ is even, the result is a knot. Now let us specify (3.1) for this particular case:

$$
\begin{aligned}
\operatorname{dim}_{q} X & =\Delta_{k} \\
A_{X Y} & =\overline{\bar{a}}_{k m} \\
\lambda_{Y}^{n_{i}} & =\bar{\lambda}_{m}^{\bar{n}_{i}}
\end{aligned}
$$


so that formula (3.1) takes the form

$$
H_{R}^{\overline{n_{1}}, \ldots, \overline{n_{g+1}}}=\sum_{k=0}^{r} \Delta_{k} \prod_{i=1}^{g+1} \sum_{m=0}^{r} \overline{\bar{a}}_{k m} \bar{\lambda}_{m}^{\bar{n}_{i}}
$$

Other cases. All other possible configurations of the pretzel links can be unified into one family with $n_{1}, \ldots, n_{2 g_{||}}$arbitrary integers associated with the parallel braids and $\overline{n_{2 g_{||}+1}}, \ldots, \overline{n_{g+1}}$ even integers associated with the antiparallel braids. Then, the constituents of (3.1) are:

$$
\begin{aligned}
& \operatorname{dim}_{q} X=\bar{\Delta}_{k} \\
& \left.\begin{array}{rl}
A_{X Y} & =a_{k m} \\
\lambda_{Y}^{n_{i}} & =\lambda_{m}^{n_{i}}
\end{array}\right\} \text { for } n_{1}, \ldots, n_{2 g_{\|}} \\
& \left.\begin{array}{rl}
A_{X Y} & =\bar{a}_{k m} \\
\lambda_{Y}^{n_{i}} & =\bar{\lambda}_{m}^{\bar{n}_{i}}
\end{array}\right\} \text { for } \overline{n_{2 g_{\mid}+1}}, \ldots, \overline{n_{g+1}},
\end{aligned}
$$

so that the answer takes the form:

$$
H_{[r]}^{n_{1}, \ldots, n_{2 g_{\|}}, \overline{n_{2 g_{\mid}+1}}, \ldots, \overline{n_{g+1}}}=\sum_{k=0}^{r} \bar{\Delta}_{k} \cdot\left\{\prod_{i=1}^{2 g_{\|}}\left(\sum_{m=0}^{r} a_{k m} \lambda_{m}^{n_{i}}\right) \cdot \prod_{j=2 g_{||}+1}^{g+1}\left(\sum_{m=0}^{r} \bar{a}_{k m} \bar{\lambda}_{m}^{\bar{n}_{j}}\right)\right\} .
$$

Thus, our formulas (3.22) and (3.24) provide the explicit answer for arbitrary pretzel link in arbitrary symmetric representation. These formulas (3.1) are perfectly consistent with (and, in fact, partly inspired by) the arbitrary genus results of [61] for the Jones polynomials. They can also be directly obtained within the framework of [63] (see section 5.5).

The HOMFLY polynomials in the totally antisymmetric representations are obtained by the usual transposition rule [37, 42]:

$$
H_{\left[1^{r}\right]}(A, q)=H_{[r]}\left(A, q^{-1}\right) .
$$

\section{Comments on the main result (3.1)}

Pretzel family. The pretzel links and knots provide us with an ample set of examples of the HOMFLY polynomials in all (anti)symmetric representations. The only examples available so far were: the Whitehead and Borromean rings links [51], the two-strand torus $[39,43]$ and twist $[44,46]$ knots parameterized by one integer each and the double braid unifying these two families and parameterized by two integer numbers [39]. These families are a tiny part of the whole pretzel family (see section 6). One of the essential points is that the pretzel family includes both thin and think [31] knots, while the twostrand torus and twist knots are all thin. The simplest example of the thick pretzel knot is $10_{139}=(4,-1,3,3)$ (see [31, eq. (49)]) in accordance with the Rolfsen tables [64]. This knot can be also obtained from knot $5_{2}$ by involving a triple braid (see [37] for details). In section 6 we list more patterns from the pretzel family. 
Torus in the t-channel $=(\overline{\mathbf{1}}, \overline{\mathbf{1}}, \overline{\mathbf{1}}, \overline{\mathbf{1}}, \overline{\mathbf{1}}, \ldots)$. The key to understanding the structure of eq. (3.22) is to note that in the particular case, when all $n_{i}=1$, we actually obtain "in the $t$-channel" the ordinary two-strand torus link $/ \operatorname{knot}: \operatorname{Pretzel}(\overline{1}, \ldots, \overline{1})=\operatorname{Torus}[2, g+1]$, i.e.

$$
H_{[r]}^{(\overline{1}, \ldots, \overline{1})}=c_{r}^{g+1} H_{[r]}^{[2, g+1]}=c_{r}^{g+1} \sum_{k=0}^{r} \Delta_{k} \lambda_{k}^{-g-1}
$$

where $c_{r}$ is a framing factor, taking into account the difference between vertical and topological framings. The "s-channel" decomposition formula in this case is

$$
H_{[r]}^{(\overline{1}, \ldots, \overline{1})}=\sum_{k=0}^{r} \Delta_{k} \prod_{j=1}^{g+1}\left(\sum_{m=0}^{r} \overline{\bar{a}}_{k m} \mu_{m}\right)
$$

which implies

$$
\sum_{m=0}^{r} \overline{\bar{a}}_{k m} \mu_{m}=c_{r} \lambda_{k}^{-1}
$$

This is, indeed, the case:

$$
\left.\frac{1+D_{1} \mu_{1}}{D_{0}}\right|_{\mu=-A}=-A q=c_{1},\left.\quad \frac{1-D_{-1} \mu_{1}}{D_{0}}\right|_{\mu=-A}=\frac{1}{A q}=c_{1} \cdot\left(-q^{-2}\right)=c_{1} \lambda_{1}^{-1}
$$

In fact, along with the orthogonality conditions (3.10), this requirement allows one to restore the whole matrix $\mathcal{A}$ in this case.

In the next paragraph we consider time-dependent quantities, thus the label $*$, referring to restriction to topological locus, which was omitted throughout the main text, is restored.

Generalizing the Rosso-Jones formula. Let us return to the Rosso-Jones formula (1.1). In the case of symmetric representations $R=[r]$, it can be written in the form

$$
\mathcal{H}_{[r]}^{[m, n]}\{p\}=q^{\frac{2 n}{m} \hat{W}_{[2]} \hat{\pi}} \chi_{[r]}\{p\}^{m}
$$

with the operator $\hat{\pi}$ changing sign of the odd character $\chi_{m}$

$$
\hat{\pi} \chi_{m}\{p\}=(-)^{m} \chi_{m}\{p\}
$$

where $m$ labels representations $Q_{m}$ arising in the two decompositions

$$
[r] \otimes[r]=\oplus_{m=0}^{r}[r+m, r-m]
$$

and

$$
[r] \otimes \overline{[r]}=\oplus_{m=0}^{r}\left[2 m, m^{N-2}\right]
$$


Clearly, our (3.24) implies an extension of (4.5) to arbitrary pretzel links/knots:

$$
\begin{aligned}
& H_{[r]}^{n_{1}, \ldots, n_{2 g_{||}}, \overline{n_{2 g_{||}+1}}, \ldots, \bar{n}_{g+1}}\{p\}= \\
& =\left(\otimes_{I=1}^{g+1} q^{n_{I} \hat{W}_{[2]}\left(p^{(I)}\right)}\right) \sum_{k=0}^{r} \bar{\Delta}_{k} \cdot\left\{\prod_{i=1}^{2 g_{\|}}\left(\sum_{m=0}^{r} \frac{a_{k m}}{\chi_{m}^{*}} \chi_{m}\left(p^{(i)}\right)\right) \cdot \prod_{j=2 g_{||}+1}^{g+1}\left(\sum_{m=0}^{r} \frac{\bar{a}_{k m}}{\bar{\chi}_{m}^{*}} \bar{\chi}_{m}\left(p^{\left(j+2 g_{\|}\right)}\right)\right)\right\} .
\end{aligned}
$$

A similar extension exists for (3.22).

Significant difference from (4.5) is that the rotation matrices $a_{k m}$ and $\bar{a}_{k m}$ depend on the representation $[r]$, and it is a challenging problem to encode this dependence into the action of some operator.

Also note that beyond the topological locus

$$
\mathcal{H}^{\left(n_{1}, n_{2}\right)}\left\{p^{(1)}, p^{(2)}\right\} \neq \mathcal{H}^{\left(n_{1}+n_{2}\right)}\{p\}
$$

— the two sides even depend on different sets of time-variables.

\section{Extension to superpolynomials and to non-symmetric representations.}

As known since [40], generalization of formulas like (3.22) and (3.24) to (anti)symmetric superpolynomials is straightforward. However, constructing the superpolynomials and another problem that can be solved by an immediate extension of these formulas, that is, constructing the HOMFLY polynomials in other representations will be considered elsewhere. Presently the best, what is known beyond arbitrary torus knots (where Rosso-Jones formula [29, 30, 38] provides generic answer in arbitrary representation) are twist knots in representation [21], see [54, 55] and, finally, [56], see also [65] for a family of torus descendants. It is (3.1) that allowed us to make a far-going conjecture [60] about a generalization of Rosso-Jones formula to all representations of genus- $g$ knots; it, however, remains to be checked. An even more challenging question is about associated generalization of the eigenvalue matrix model (1.4), currently it is available only for twist knots $[52,53]$.

$\boldsymbol{A}$-polynomials. One can study the dependence of the constructed HOMFLY polynomials (3.22) and (3.24) on spin of the representation (or representations in the case of links). One of the ways to describe this dependence is to derive difference equations with respect to the spin variables. There are various types of these relations [28], some of them are very easy to observe, other ones are usually much more complicated but instead they can be related to the volume conjecture [66-69] and their "quasiclassical" limit is given by the $A$-polynomial [70-73] (the so-called AJ-conjecture) and, for this reason, the equations are called "quantum $A$-polynomials". They can be found with the help of computer programs implementing Zeilberger's algorithm for the hypergeometric sums [74, 75]. Since the HOMFLY polynomials in any symmetric representations were known so far only for a few cases (see above), only in those cases the quantum $A$-polynomial was calculated. Our results (3.22) and (3.24) open a road for obtaining many more $A$-polynomials, though these expressions literally are not suitable and still have to be reshuffled: they have no form of a $q$ hypergeometric polynomial and the existing software implementing Zeilberger's algorithm for the hypergeometric sums $[74,75]$ can not be immediately used. 


\section{$5 \quad$ Matrices $a_{k m}$ and $\bar{a}_{k m}$ as universal Racah matrix}

In the case of Jones polynomials (i.e., for $A=q^{2}$ ), the simplest matrix $\mathcal{A}$ turns into

$$
\mathcal{A}_{1}=\left(\begin{array}{ll}
1 & {[3]} \\
1 & -1
\end{array}\right)
$$

and an immediate desire is to compare it with the celebrated fusion (mixing) matrix

$$
S=\frac{1}{[2]}\left(\begin{array}{cc}
1 & \sqrt{[3]} \\
\sqrt{[3]} & -1
\end{array}\right)
$$

which recently appeared in many places, from modular transformation of the simplest Virasoro conformal block in [76] to elementary three-strand knot calculations in [12]. This similarity turned out to be not a simple coincidence, but a manifestation of general fact: in full generality the matrices $\mathcal{A}$ in our formulas for the genus- $g$ knot polynomials are nothing but a simple rescaling of the Racah matrices from representation theory of quantum groups, of which $S$ is just the simplest example. This fact, what came for us as a result of tedious calculations, was announced in a separate paper [60]. Though this is nearly obvious after being discovered and is spectacularly confirmed by the derivation of eqs. (3.8)-(3.10), in this section we provide a little more details and comments.

First of all, in variance with the Jones case, where the relevant group is $S U_{q}(2)$ and the Racah matrices are long known from [77-79], in the HOMFLY case one needs generic $S U_{q}(N)$ matrices $\mathcal{A}$ which depend on $A$. Therefore, they are universal objects, interpolating between the Racah matrices for particular $S U_{q}(N)$ at $A=q^{N}$. Not much was known about such quantities until recently, fortunately, the very recent [80] provides the needed information. Second, in the HOMFLY case the set of allowed representations is wider than that in the Jones case: even if one restricts considerations to symmetric representations, their conjugates unavoidably enter the game, and they are no longer the same, as they were for $S U_{q}(2)$.

Having this said, let us return to our main formula (3.1) which was conjectured in $[60]$ yet

$$
H_{R}^{n_{1}, \ldots, n_{g+1}} \quad=\sum_{\mathcal{X}} \operatorname{dim}_{q} \mathcal{X} \prod_{i=1}^{g+1} \sum_{\mathcal{Y}} \mathcal{A}_{\mathcal{X} Y} \lambda_{\mathcal{Y}}^{n_{i}}
$$

and comment on it in a little more detail. In fact, this formula naturally generalizes to $l$ different representations in the case of $l$-component link [60] (see figure 6).

1. In this formula, one can understand under the calligraphic index either $X$ or $\bar{X}$ and similarly for $\mathcal{Y}$ so that $\bar{\Delta}_{X} \equiv \Delta_{\bar{X}}, \bar{\lambda}_{X} \equiv \lambda_{\bar{X}}$ etc. Then, there are three possibilities: when in (5.3) enter $X$ and $\bar{Y}, \bar{X}$ and $\bar{Y}, \bar{X}$ and $Y$. Accordingly, there are three different matrices $\mathcal{A}_{X \bar{Y}}, \mathcal{A}_{\bar{X} \bar{Y}}$ and $\mathcal{A}_{\bar{X} Y}$ which correspond to (3.8), (3.9) and (3.10).

2. The three orthogonality conditions (3.15)-(3.18) satisfied by the matrices $\mathcal{A}$ can be rewritten in these terms as the single equation

$$
\sum_{\mathcal{X}} \operatorname{dim}_{q} \mathcal{X} \cdot \mathcal{A}_{\mathcal{X Y}} \cdot \mathcal{A}_{\mathcal{X} \mathcal{Y}^{\prime}}=\operatorname{dim}_{q} \mathcal{Y} \delta \mathcal{Y}, \mathcal{Y}^{\prime}
$$


and similarly for the dual one (3.16). This means that the relation to the orthonormal Racah matrix $S$ is

$$
\mathcal{A}_{\mathcal{X Y}}=\sqrt{\frac{\operatorname{dim}_{q} \mathcal{Y}}{\operatorname{dim}_{q} \mathcal{X}}} S_{\mathcal{X Y}}
$$

After such a rescaling our formulas (3.8) and (3.9) seem to be in a perfect agreement with the conjectures of [80], thus justifying/supporting our suggested identification of $\mathcal{A}$ as the rescaled Racah matrices.

3. Note that there are exactly three possible Racah matrices when all the representations are either $R$ or $\bar{R}$ : $S\left(\begin{array}{ll}R & \bar{R} \\ R & \bar{R}\end{array}\right), S\left(\begin{array}{ll}\bar{R} & \bar{R} \\ R & R\end{array}\right)$ and $S\left(\begin{array}{ll}\bar{R} & R \\ R & \bar{R}\end{array}\right)$.

These three cases correspond to the three types of the matrices $\mathcal{A}$ discussed above: $\mathcal{A}_{X \bar{Y}}, \mathcal{A}_{\bar{X} Y}$ and $\mathcal{A}_{\bar{X} \bar{Y}}$. The first two are transposed to each other (by the general properties of the Racah matrices) and the third one is symmetric. This also explains why the factor $\overline{\mathcal{G}}$ in (3.14) is symmetric in $k$ and $m$, while $\mathcal{G}$ is not: in the latter case $k$ and $m$ label different representations, $k \in[r] \otimes \overline{[r]}, m \in[r] \otimes[r]$, while in the former case both $k, m \in[r] \otimes \overline{[r]}$.

4. One can make use of the additional fact that the first line of the matrices $\mathcal{A}_{\mathcal{X Y}}$, associated with the singlet representation $X=\bar{\emptyset}$, consists just of the quantum dimensions $\chi \mathcal{Y}=\operatorname{dim}_{q} \mathcal{Y},(3.19),(3.20)$ and rewrite (5.3) through the orthonormal Racah matrix $S$ in another form:

$$
H_{R}^{n_{1}, \ldots, n_{g+1}}=\sum_{\mathcal{X}}\left(\operatorname{dim}_{q} \mathcal{X}\right)^{\frac{1-g}{2}} \prod_{i=1}^{g+1}\left(\sum_{\mathcal{Y}} S_{\mathcal{X} \mathcal{Y}} S_{\bar{\emptyset} \mathcal{Y}} \lambda_{\mathcal{Y}}^{n_{i}}\right)
$$

In result, the contributions of parallel, even antiparallel and odd antiparallel braids are respectively $S_{\bar{X} Y} S_{\bar{\emptyset} Y}, S_{\bar{X} \bar{Y}} S_{\bar{\emptyset} \bar{Y}}$ and $S_{X \bar{Y}} S_{\bar{\emptyset} \bar{Y}}$. In the case of $g=1$ (torus knots/links) the factor $\operatorname{dim}_{q} \mathcal{X}$ is absent and one can sum over $\mathcal{X}$, using the orthonormality condition $\sum_{\mathcal{X}} S_{\mathcal{X} Y} S_{\mathcal{X} \mathcal{Y}^{\prime}}=\delta_{\mathcal{Y} \mathcal{Y}^{\prime}}$, to get just the Rosso-Jones formula in the form of [61]

$$
H^{\left(n_{1}, n_{2}\right)}=\sum_{\mathcal{Y}} S_{\emptyset \mathcal{Y}}^{2} \lambda_{\mathcal{Y}}^{n_{1}+n_{2}}
$$

The structure of (5.6), involving a sum with a weight, which is the power $2-2 g$ (the Euler characteristics of the genus $g$ Riemann surface) of representation dependent quantity resembles the Frobenius formula [81], typical for topological (cohomological) models.

5. Note that formula (5.6) can be immediately obtained from the conjectural representation proposed in [63], which generalizes the approach due to E.Witten [27]. E.Witten interpreted the averages of Wilson lines in Chern-Simons theory within the framework of a Hamiltonian dynamics on the space of conformal blocks in the WZWN model 


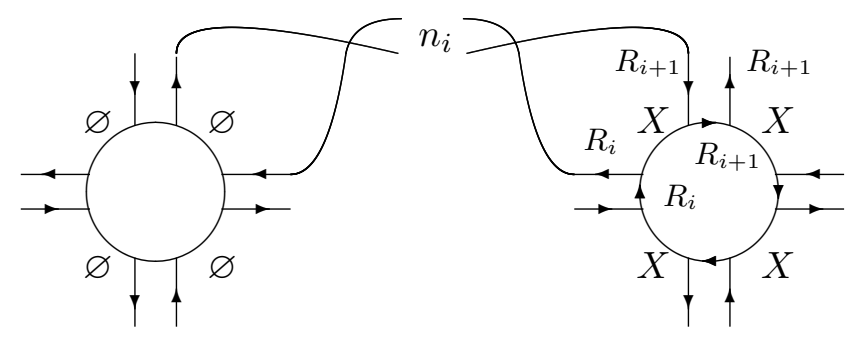

Figure 6. Conformal block representation of formula (5.6). Here the generic case of different representations $R_{i}$ (in the case of link) is drawn, it corresponds to $\sum_{\bar{X} \in \bigcap_{i} R_{i} \otimes \bar{R}_{i}} \operatorname{dim}_{q} \bar{X}$. $\prod_{i=0}^{g}\left(\sum_{\bar{Y}_{i} \in R_{i} \otimes \bar{R}_{i+1}} \mathcal{A}_{\bar{X}_{\bar{Y}} \bar{Y}_{i}}\left(\begin{array}{cc}\bar{R}_{i} R_{i+1} \\ R_{i} & \bar{R}_{i+1}\end{array}\right) \cdot \lambda_{\bar{Y}_{i}}^{n_{i}}\right)$, or other two matrices $\mathcal{A}$ depending on the direction of arrows in the picture.

with evolution given by permutations of points of these conformal blocks. One can calculate in this way knot invariants in terms of matrices of modular transformations of conformal blocks [13-18]. In [63] (see also earlier paper [82]), the authors proposed to generalize this case describing the Hamiltonian evolution of a conformal block as an "in"-state to another conformal block as an "out"-state to the case of a few sets of states "at infinity", i.e. to the case of a few boundaries in terms of Chern-Simons theory. However, their conjecture could be checked so far only in the fundamental representation for many knots with less than 11 intersections in the Rolfsen tables [64] and for some knots in first few symmetric representations [63] (very recently it was further checked in representation [21], see [83]). Now note that the pretzel knots can be drawn by gluing of $\mathrm{v} 5$ and $\mathrm{v} 6$ (or $\mathrm{v} 7$ and $\mathrm{v} 8$ ) repeatedly in figure 3 of [63], which will naturally provide the formula (62). Hence, to put our results for the pretzel knots here differently, being obtained basically within the absolutely different and well established group theory approach [1-11] they can be considered as a first confirmation of the conjecture [63] for the infinite series of knots and infinite series of representations.

6. At a deeper level, the relation of (5.6) to the conformal block calculus of [13-18] and [61] remains a mystery. It can be schematically realized with a toric conformal block picture, see figure 6 . The occurrence of the toric blocks can seem natural for the pretzel family, but exact appearance, and the very possibility to derive (5.6) from consideration of the spherical blocks, as done in [61] implies some interrelation in the style of Verlinde formulas, which needs to be put in a more precise form.

7. For generic representations one has: $R_{1} \otimes R_{2}=\oplus X \otimes V_{X}$. When the multiplicities of all $X$ are unities, as in the case of a product of two symmetric representations and/or of their conjugates, $\operatorname{dim}_{q} X$ is just a number, dimension of the representation $X$. In this case, (5.6) is symmetric under arbitrary permutations of $n_{i}$ (if all $R_{i}$ are the same), i.e. there is the enhanced symmetry. However, when multiplicity of $X$ is nontrivial, i.e. $V_{X}$ is a vector space of non-unit dimension, then the matrix $\mathcal{A}_{X Y}$ is a vector in $V_{X}$ and $\operatorname{dim}_{q} X$ in (5.6) is rather a multi-linear operation $V_{X}^{\otimes(g+1)} \longrightarrow 1$, which 
does not need to be totally symmetric: only the cyclic symmetry is needed. This is in accordance with the fact that a non-cyclic permutation of $n_{i}$ converts a knot/link into a mutant, undistinguishable by symmetrically-colored knot polynomials, but separable by those in non-(anti)symmetric representations.

\section{Checks of the main conjecture (3.1)}

So far there were just two families of knots with explicitly known (anti)symmetric HOMFLY polynomials: torus knots [29, 30, 37] and double braids [39] (and also a family of links [51]). It is this second family that we enormously extend in the present paper. The double braids are Pretzel $:(-1, \overline{2 k}, n)=(\bar{n}, \underbrace{\overline{1}, \ldots, \overline{1}}_{2 k})$ and contain twist knots $(n=-1)$, in particular the figure-eight knot $4_{1}$, which was the first example beyond torus knots, studied in [40]. Our formulas are of course consistent with the results of [29, 30, 37] and [39], but [39] and [41] contains more: differential expansions, which still needs to be studied in generic pretzel case.

Below we list some tests which we did to examine our main conjecture (3.1). Also we tabulate some knots and links which belong to the pretzel family.

\subsection{Jones polynomials}

Technically, the simplest way of checking/deriving the main conjecture is to use the physically motivated calculations via conformal blocks of either WZWN theory [13-18] or of the minimal models [76]. The case of Jones polynomials, which are obtained from (3.1) at specialization of $A=q^{2}$, is much easier to study technically, it was done in [60,61] and the obtained result for an arbitrary pretzel knot/link completely agrees with (3.1) at $A=q^{2}$.

\subsection{Comparing with families of knots/links}

For some particular examples we have checked that our formula (3.1) reproduces the HOMFLY polynomials in symmetric representations:

\section{Knots:}

- twist knots $[39,40,44,46]: T^{(k)}=(\overline{1}, \overline{1}, \overline{2 k-1})=(-1,-1, \overline{2 k})$ (we remind that $T^{(-k)}=(2 k+2)_{1}$ for $k>0, T^{0}=$ unknot, $T^{(1)}=3_{1}, T^{(k)}=(2 k+1)_{2}$ for $\left.k>1\right)$;

- torus knots and links $[39,43]:[2, n]=(n, 0)=(1, n-1),[3,4]=8_{19}=(3,3, \overline{-2})$, $[3,5]=(5,3, \overline{-2})$, all other are not pretzel knots.

These examples all belong to the larger family:

- double braid [39]: $(-1, \overline{2 k}, n)=(\bar{n}, \underbrace{\overline{1}, \ldots, \overline{1}}_{2 k})$.

In this case, the HOMFLY polynomials are known for all symmetric representations, and it completely describes the knots in the parallel-anti-parallel case in genus two. 


\section{Links:}

- Whitehead link [51] (in this case two different representations are allowed on the two different components of the link): $L_{5} a_{1}=(2, \overline{2}, 1)$.

This link is also a member of a whole family of

- links [51]: $(2, \overline{2 m}, 1)$ : Hopf, Whitehead, L7a6, L9a36, L11a360.

The HOMFLY polynomials for the family are known only when one of the representations is arbitrary symmetric. We checked the Whitehead link with two coinciding representations on each component of link being any symmetric representation, and the whole family with the fundamental representations only.

The answers in these case, both for knots and links coincide with the prediction of formula (3.1).

\subsection{Comparing with arbitrary pretzels at concrete representations}

We also checked the main conjecture at comparing the answers obtained by the evolution method [39] for arbitrary pretzel knots: of genus 2 and representations [1], [2], [3]; of genus 3,4 and representations [1], [2]; of genus up to 9 and the fundamental representation. The formulas are quite tedious, some part of them can be found in appendix A.

\subsection{Reduction and expansion tests}

There are a few tests that the answers for the HOMFLY polynomials should pass, which allowed us to additionally test our main conjecture (3.1).

1. Special polynomials $H_{R}^{\mathcal{K}}(A, q=1)$ provided a very good test due to the factorization property [37, 84]:

$$
\sigma_{R}(A)=\lim _{q \longrightarrow 1} \frac{H_{R}(q, A)}{\chi_{R}(q, A)}=\left(\sigma_{[1]}(A)\right)^{|R|}
$$

2. Alexander polynomials $H_{R}^{\mathcal{K}}(A=1, q)$ also have a simple representation dependence for hook diagrams which include symmetric representations [40]

$$
\frac{H_{R}}{\chi_{R}}(A=1, q)=\frac{H_{[1]}}{\chi_{[1]}}\left(A=1, q^{|R|}\right)
$$

and also provided a very good test for our results.

3. Another powerful tool to test the HOMFLY polynomials in any representation, which we used here, is expansion through the Vassiliev invariants and trivalent diagrams. We shall not explain this expansion here and refer our readers to the literature [85-87]. 


\subsection{Table: Rolfsen and Thistlethwaite vs. pretzel [64]}

We checked that our main conjecture gives the correct results for all fundamental HOMFLY polynomials for the pretzel knots with up to 10 crossings (plus mutant pairs with 11 crossings). Note that our conjecture was recently tested even in a more general than pretzel set of knots in representations up to the first mixed one [21], [83].

Understanding if the given knot/link belongs to the pretzel family is not quite a trivial exercise. However, since we now possess generic expression for pretzel symmetric HOMFLY, this can be done systematically, by comparing our results with the polynomials in [64] (note that there is just one coincidence between fundamental HOMFLY for up-to-ten-crossings knots - for $5_{1}$ and $10_{132}$ ). Result of this analysis is the following list. We use condensed notation $1^{5}=1,1,1,1,1$ and also do not distinguish between knots and their mirrors, when the signs in all crossings are reversed. Symbol "wn" stands for knot mutation.

\begin{tabular}{|c|c|c|c|c|c|c|c|c|c|}
\hline \multicolumn{10}{|c|}{ Knots, \#crossings $=\mathbf{3} \mathbf{. 8}$} \\
\hline $\mathbf{3}_{\mathbf{1}}$ & $(3,0)$ & $\mathbf{7}_{\mathbf{1}}$ & $(7,0)$ & $\mathbf{8}_{\mathbf{1}}$ & $(1, \overline{6}, 1)$ & $\mathbf{8}_{\mathbf{8}}$ & $(2,-3,1,1,1,1)$ & $\mathbf{8}_{\mathbf{1 5}}$ & $(2,3,3,-1,-1,-1)$ \\
\hline $\mathbf{4}_{\mathbf{1}}$ & $(1, \overline{2}, 1)$ & $\mathbf{7}_{\mathbf{2}}$ & $(\overline{5}, \overline{1}, \overline{1})$ & $\mathbf{8}_{\mathbf{2}}$ & $(5, \overline{2}, 1)$ & $\mathbf{8}_{\mathbf{9}}$ & $(4,-3,-1,-1)$ & $\mathbf{8}_{\mathbf{1 6}}$ & \\
\hline $\mathbf{5}_{\mathbf{1}}$ & $(5,0)$ & $\mathbf{7}_{\mathbf{3}}$ & $(4,1,1,1)$ & $\mathbf{8}_{\mathbf{3}}$ & $(1,1, \overline{4}, 1,1)$ & $\mathbf{8}_{\mathbf{1 0}}$ & $(2,-3,1,3)$ & $\mathbf{8}_{\mathbf{1 7}}$ & \\
\hline $\mathbf{5}_{\mathbf{2}}$ & $(\overline{3}, \overline{1}, \overline{1})$ & $\mathbf{7}_{\mathbf{4}}$ & $(\overline{3}, \overline{3}, \overline{1})$ & $\mathbf{8}_{\mathbf{4}}$ & $(3, \overline{4}, 1)$ & $\mathbf{8}_{\mathbf{1 1}}$ & $(-\overline{3}, \overline{1}, \overline{1}, \overline{3}, \overline{1})$ & $\mathbf{8}_{\mathbf{1}}$ & \\
\hline $\mathbf{6}_{\mathbf{1}}$ & $(\overline{5},-\overline{1},-\overline{1})$ & $\mathbf{7}_{\mathbf{5}}$ & $(3,2,1,1)$ & $\mathbf{8}_{\mathbf{5}}$ & $(3, \overline{2}, 3)$ & $\mathbf{8}_{\mathbf{1 2}}$ & & $\mathbf{8}_{\mathbf{1 9}}$ & $(3,-\overline{2}, 3)$ \\
\hline $\mathbf{6}_{\mathbf{2}}$ & $(3, \overline{2}, 1)$ & $\mathbf{7}_{\mathbf{6}}$ & $(-3,1, \overline{2}, 1,1)$ & $\mathbf{8}_{\mathbf{6}}$ & $(1,3, \overline{2}, 1,1)$ & $\mathbf{8}_{\mathbf{1 3}}$ & $(-\overline{4},-3,1,1,1)$ & $\mathbf{8}_{\mathbf{2 0}}$ & $(3, \overline{2},-3)$ \\
\hline $\mathbf{6}_{\mathbf{3}}$ & $(2,-3,1,1)$ & $\mathbf{7}_{\mathbf{7}}$ & $(-\overline{3}, \overline{1},-\overline{3}, \overline{1}, \overline{1})$ & $\mathbf{8}_{\mathbf{7}}$ & $(4,-3,1,1)$ & $\mathbf{8}_{\mathbf{1 4}}$ & & $\mathbf{8}_{\mathbf{2 1}}$ & $(2,-3,1,-3)$ \\
\hline \multicolumn{70}{|c|}{ Links } \\
\hline $\mathbf{L}_{\mathbf{2}} \mathbf{a}_{\mathbf{1}}$ & $(2,0)$ & $\mathbf{L}_{\mathbf{6}} \mathbf{a}_{\mathbf{1}}$ & $(2,1,1,2)$ & $\mathbf{L}_{\mathbf{6}} \mathbf{a}_{\mathbf{4}}$ & $?$ & & & \\
\hline $\mathbf{L}_{\mathbf{4}} \mathbf{a}_{\mathbf{1}}$ & $(4,0)$ & $\mathbf{L}_{\mathbf{6}} \mathbf{a}_{\mathbf{2}}$ & $(3,1,1,1)$ & $\mathbf{L}_{\mathbf{6}} \mathbf{a}_{\mathbf{5}}$ & $(2, \overline{2}, 2)$ & & & \\
\hline $\mathbf{L}_{\mathbf{5}} \mathbf{a}_{\mathbf{1}}$ & $(2, \overline{2}, 1)$ & $\mathbf{L}_{\mathbf{6}} \mathbf{a}_{\mathbf{3}}$ & $(6,0)$ & $\mathbf{L}_{\mathbf{6}} \mathbf{n}_{\mathbf{1}}$ & $(-2,-\overline{\mathbf{2}}, 2)$ & & & & \\
\hline
\end{tabular}

\begin{tabular}{|c|c|c|c|c|c|c|c|c|c|}
\hline \multicolumn{10}{|c|}{ Knots, \#crossings $=9$} \\
\hline $9_{1}$ & $(9,0)$ & $9_{11}$ & $\left(-5,-2,1^{4}\right)$ & $9_{21}$ & & $9_{31}$ & & $9_{41}$ & \\
\hline $9_{2}$ & $(\overline{1}, \overline{7}, \overline{1})$ & $9_{12}$ & $(-3,1,1,1, \overline{4})$ & $9_{22}$ & & $9_{32}$ & & $9_{42}$ & \\
\hline $9_{3}$ & $(6,1,1,1)$ & $9_{13}$ & $(1,3,1,1,-\overline{4})$ & $9_{23}$ & & $9_{33}$ & & $9_{43}$ & \\
\hline $9_{4}$ & $\left(4,1^{5}\right)$ & $9_{14}$ & $(-\overline{5},-\overline{3}, \overline{1}, \overline{1}, \overline{1})$ & $9_{24}$ & $\left(-2,-3,3,1^{3}\right)$ & $9_{34}$ & & $9_{44}$ & \\
\hline $9_{5}$ & $(-\overline{1},-\overline{3},-\overline{5})$ & $9_{15}$ & & $9_{25}$ & & $9_{35}$ & $(\overline{3}, \overline{3}, \overline{3})$ & $9_{45}$ & \\
\hline $9_{6}$ & $(2,1,5,1)$ & $9_{16}$ & $(\overline{2}, 1,3,3)$ & $9_{26}$ & & $\mathbf{9}_{36}$ & & $9_{46}$ & $(\overline{3},-\overline{3}, \overline{3})$ \\
\hline $9_{7}$ & $\left(2,3,1^{4}\right)$ & $9_{17}$ & $\left(\overline{3}, \overline{3},-\overline{1}^{5}\right)$ & $9_{27}$ & & $9_{37}$ & $(-\overline{3},-\overline{3}, \overline{3}, \overline{1}, \overline{1})$ & $9_{47}$ & \\
\hline $9_{8}$ & $\left(-2,-3,1^{6}\right)$ & $9_{18}$ & & $9_{28}$ & $\left(2,-3,-3,1^{3}\right)$ & $9_{38}$ & & $9_{48}$ & $(-\overline{3},-\overline{3},-\overline{3}, \overline{1}, \overline{1})$ \\
\hline 99 & $(-4,1,-5,1)$ & $9_{19}$ & & $9_{29}$ & & $9_{39}$ & & $9_{49}$ & \\
\hline $9_{10}$ & $(\overline{3}, \overline{3}, \overline{1}, \overline{1}, \overline{1})$ & $9_{20}$ & $\left(4,3,-1^{4}\right)$ & $9_{30}$ & & $9_{40}$ & & & \\
\hline
\end{tabular}




\begin{tabular}{|c|c|c|c|c|c|c|c|}
\hline \multicolumn{7}{|c|}{ Knots, \#crossings = 10 } \\
\hline $\mathbf{1 0}_{\mathbf{1}}$ & $(\overline{1}, \overline{7},-\overline{3})$ & $\mathbf{1 0}_{\mathbf{1 6}}$ & $(\overline{3}, \overline{1},-\overline{5}, \overline{1}, \overline{1})$ & $\mathbf{1 0}_{\mathbf{6 1}}$ & $(3,3, \overline{4})$ & $\mathbf{1 0}_{\mathbf{1 2 6}}$ & $(\overline{2},-5,3) \bigvee(-2,3,-5,1)$ \\
\hline $\mathbf{1 0}_{\mathbf{2}}$ & $(2,-7,-1,-1)$ & $\mathbf{1 0}_{\mathbf{1 7}}$ & $(4,-5,1,1)$ & $\mathbf{1 0}_{\mathbf{6 2}}$ & $(4,-3,1,3)$ & $\mathbf{1 0}_{\mathbf{1 2 7}}$ & $(\overline{2}, 5,3) \bigvee(2,-5,-3,1)$ \\
\hline $\mathbf{1 0}_{\mathbf{3}}$ & $(\overline{1}, \overline{5},-\overline{5})$ & $\mathbf{1 0}_{\mathbf{1 9}}$ & $(\overline{4}, 5,-1,-1,-1)$ & $\mathbf{1 0}_{\mathbf{6 3}}$ & $(\overline{4},-3,-3,1,1)$ & $\mathbf{1 0}_{\mathbf{1 2 9}}$ & $(2,1,1,-3,1,1)$ \\
\hline $\mathbf{1 0}_{\mathbf{4}}$ & $(-\overline{7}, \overline{1}, \overline{1}, \overline{1}, \overline{1})$ & $\mathbf{1 0}_{\mathbf{2 0}}$ & $\left(-2,1,3,1^{5}\right)$ & $\mathbf{1 0}_{\mathbf{6 4}}$ & $(-4,3,3,1)$ & $\mathbf{1 0}_{\mathbf{1 3 9}}$ & $(4,-1,3,3)$ \\
\hline $\mathbf{1 0}_{\mathbf{5}}$ & $(-2,7,-1,-1)$ & $\mathbf{1 0}_{\mathbf{2 1}}$ & $\left(-\overline{3}, \overline{3}, \overline{1}^{5}\right)$ & $\mathbf{1 0}_{\mathbf{6 5}}$ & $(\overline{4}, 3,-3,-1,-1)$ & $\mathbf{1 0}_{\mathbf{1 4 0}}$ & $(-3,3, \overline{4})$ \\
\hline $\mathbf{1 0}_{\mathbf{6}}$ & $\left(-\overline{2},-5,-1^{3}\right)$ & $\mathbf{1 0}_{\mathbf{2 2}}$ & $(-4,1,1,3,1,1)$ & $\mathbf{1 0}_{\mathbf{6 9}}$ & $(4,3,3,-1,-1,-1)$ & $\mathbf{1 0}_{\mathbf{1 4 1}}$ & $(4,-3,-3,1)$ \\
\hline $\mathbf{1 0}_{\mathbf{7}}$ & $(-\overline{3}, \overline{1}, \overline{5}, \overline{1}, \overline{1})$ & $\mathbf{1 0}_{\mathbf{2 8}}$ & $\left(\overline{4}, 3,-1^{5}\right)$ & $\mathbf{1 0}_{\mathbf{7 4}}$ & $(-\overline{3}, \overline{1}, \overline{3}, \overline{3}, \overline{1})$ & $\mathbf{1 0}_{\mathbf{1 4 2}}$ & $(3,3,-\overline{4})$ \\
\hline $\mathbf{1 0}_{\mathbf{8}}$ & $\left(-6,1^{5}\right)$ & $\mathbf{1 0}_{\mathbf{3 4}}$ & $\left(2,-3,1^{6}\right)$ & $\mathbf{1 0}_{\mathbf{7 6}}$ & $(1,3,3,1, \overline{2})$ & $\mathbf{1 0}_{\mathbf{1 4 3}}$ & $(-4,3,1,-3)$ \\
\hline $\mathbf{1 0}_{\mathbf{9}}$ & $(6,-3,-1,-1)$ & $\mathbf{1 0}_{\mathbf{4 6}}$ & $(-2,3,5,1)$ & $\mathbf{1 0}_{\mathbf{7 7}}$ & $(2,-3,1,3,1,1)$ & $\mathbf{1 0}_{\mathbf{1 4 4}}$ & $(\overline{4}, 3,3,-1,-1)$ \\
\hline $\mathbf{1 0}_{\mathbf{1 1}}$ & $(3,1,1,1, \overline{4})$ & $\mathbf{1 0}_{\mathbf{4 7}}$ & $(2,-3,5,1)$ & $\mathbf{1 0}_{\mathbf{7 8}}$ & $(\overline{2},-3,-3,1,1,1,1)$ & & \\
\hline $\mathbf{1 0}_{\mathbf{1 2}}$ & $(4,-3,1,1,1,1)$ & $\mathbf{1 0}_{\mathbf{4 8}}$ & $(2,-5,1,3)$ & $\mathbf{1 0}_{\mathbf{1 2 4}}$ & $(\overline{2},-5,-3) \bigvee(2,-1,5,3)$ & & \\
\hline $\mathbf{1 0}_{\mathbf{1 5}}$ & $\left(-2,-1,5,-1^{3}\right)$ & $\mathbf{1 0}_{\mathbf{4 9}}$ & $(\overline{2},-5,-3,1,1)$ & $\mathbf{1 0}_{\mathbf{1 2 5}}$ & $(\overline{2}, 5,-3) \bigvee(2,-5,-1,3)$ & & \\
& & & $\left(2,5,3,-1^{3}\right)$ & & & & \\
\hline
\end{tabular}

\begin{tabular}{|c|c|c|c|c|}
\hline \multicolumn{5}{|c|}{ Mutants, \#crossings $=11$} \\
\hline $11_{44}^{\mathrm{a}}$ & $(-3,3,2,1,1,-3)$ & $m \rightarrow$ & $11_{47}^{\mathrm{a}}$ & $(3,-3,2,1,1,-3)$ \\
\hline $11_{57}^{\mathrm{a}}$ & $(\overline{2}, 1,3,3,-3)$ & $m$ & $11_{231}^{\mathrm{a}}$ & $(\overline{2}, 1,3,-3,3)$ \\
\hline $11_{71}^{\mathrm{n}}$ & $(\overline{2},-3,3,-3,1)$ & $\leftrightarrow$ & $11_{75}^{n}$ & $(\overline{2}, 3,-3,-3,1)$ \\
\hline $11_{73}^{\mathrm{n}}$ & $(2,3,-3,-3)$ & $\leftrightarrow$ & $11_{74}^{\mathrm{n}}$ & $(2,-3,3,-3)$ \\
\hline $11_{76}^{\mathrm{n}}$ & $(2,3,3,-3)$ & $m \rightarrow$ & $11_{78}^{\mathrm{n}}$ & $(2,3,-3,3)$ \\
\hline
\end{tabular}

\subsection{Generalizations}

As already mentioned, the results of the present paper were recently extended in [83] in two directions: to a wide class of non-pretzel knots and to the first non-(anti)symmetric representation [21]. This provides a lot of additional tests, from matching with increased number of previously known HOMFLY polynomials to comparing with the celebrated results due to H. Morton, P. Cromwell [88] and J. Murakami [89] for the mutant pairs of knots. We consider this new development as one more, and very convincing confirmation of the main conjecture (3.1).

\section{Conclusion}

In this paper we reported the results about the HOMFLY polynomials for the pretzel knots, which are a natural generalization of the torus knots from $g=1$ to arbitrary genus $g$, for which an exhaustive answer like the Rosso-Jones formula can presumably be found.

Indeed, we found a well-structured exhaustively explicit answer for arbitrary $g$ in all (anti)symmetric representations, and indeed the Rosso-Jones formula arises as its very special case. Not surprisingly, this general answer involves more than just quantum dimensions, but also the Racah matrices, however, in the absolutely minimal way which agrees with the approach proposed in [63]. As a byproduct of our calculation, an explicit formula for Racah matrices was found in symmetric representations, which is completely in 
accord with the recent result in [80]. A stronger conjecture about generic representations, formulated in [60] on the base of the present paper, needs more work to be checked. In an accompanying paper [61] some parallel evidence is obtained by different method for the Jones polynomials: that work served as a major inspiration for some of the above calculations.

Further work in this direction seems to be very promising and can lead to considerable extension of the set of known knot polynomials. An absolutely new kind of decomposition of knot polynomials into the Racah rotated elementary HOMFLY, as well as emerging a partly expected connection to the (modular transformations of the) toric conformal blocks requires better understanding, perhaps, as a kind of monopole/brane duality, and suggests various generalizations and implications. All this can open a new intriguing chapter in the theory of knot polynomials.

There are five obvious exercises to do, once the full evolution induced answer is known:

(i) to derive differential expansions a la [40,41],

(ii) to find equations w.r.t. the $r$-parameter [28, 70-73],

(iii) to study the large-r (Kashaev or volume conjecture) limit [66-69],

(iv) to use it to built a matrix model a la $[52,53]$,

(v) to perform $\beta$-deformation [90] a la [37], [91] and [40, 43, 44, 46], i.e. to promote HOMFLY to superpolynomials without explicit application of the sophisticated KhovanovRozansky construction [92-94] and even of its simplified modern substitutes [95, 96].

Also straightforward should be generalization to various extensions of the pretzel family, like combinations of multi-strand braids and their "iterations" a la [65]. These considerations will be reported elsewhere.

A really difficult task is going from (anti)symmetric to generic representations. If one believes that the conjecture (5.3) or something similar holds for them, the problem is actually about the generic Racah matrices, which is a kind of a classical hard problems in group theory. Still, as the results in the present paper demonstrate once again, study of the knot polynomials can provide a new powerful tool to attack such old problems: after the answer for the generic symmetric Racah coefficients appeared very easily in this way, one can anticipate insights about other representations as well. For some yet-nonsystematic considerations of non-symmetric colored knot polynomials beyond the torus links see $[45,54-56] .^{2}$

\section{Acknowledgments}

We would like to thank A. Anokhina, S. Arthamonov, D. Galakhov, D. Melnikov and And. Morozov for fruitful discussions.

\footnotetext{
${ }^{2}$ For an impressive progress in this direction in development of the present paper see [83] and comments in the newly added section 6.6 above.
} 
Our work is partly supported by grants NSh-1500.2014.2, by RFBR grants 13-02-00457 (A.Mir. \& A.S.), 13-02-00478 (A.Mor.), by the joint grants 13-02-91371-ST-a, 15-51-52031-NSC-a, by 14-01-92691-Ind-a. Also we are partly supported by the Brazilian National Counsel of Scientific and Technological Development (A.Mor.), by foundation FUNPEC-UFRN (A.Mir. \& A.S.), and by the Quantum Topology Lab of Chelyabinsk State University (Russian Federation government grant 14.Z50.31.0020) (A.S.).

\section{A Symmetrically colored HOMFLY for generalized pretzel knots}

Here we present a few sample results, obtained by direct application of evolution method of [37] (see [39] for the detailed explanation). They are the origin and justification of all the results in the main text. Other numerous explicit formulas of this kind are too big to be included here, still these examples are sufficient - but only for illustrative purposes. When some properties, like enhanced symmetry w.r.t. arbitrary permutations of $n_{i}$ among parallel or antiparallel braids, are mentioned in the main text, they were actually obtained from explicit evolution-method calculations - not actually represented in this appendix. After established in simpler examples, these properties were used as input assumption in more complicated ones, thus allowing to decrease the number of requested "initial conditions" - still some random checks of these assumptions were also performed at these next levels of complexity.

Summary, genus $\mathbf{2}, \boldsymbol{n}_{\mathbf{2}}$ even. In this case there is one obvious structure: for $n_{2}=0$ we obtain a composite knot made from two 2-strand torus knots.

Unreduced colored HOMFLY in the lowest symmetric representations are:

$$
\begin{aligned}
\chi_{[1]} H_{[1]}^{\left(n_{1}, \overline{n_{2}}, n_{3}\right)}= & \lambda_{[2]}^{n_{1}+n_{3}} \cdot \chi_{[2]}\left\{1+\frac{D_{2} D_{-1}}{[2]} \cdot A^{n_{2}}\right\}+ \\
& +\lambda_{[11]}^{n_{1}+n_{3}} \cdot \chi_{[11]}\left\{1+\frac{D_{1} D_{-2}}{[2]} \cdot A^{n_{2}}\right\}+\left(\lambda_{[2]}^{n_{1}} \lambda_{[11]}^{n_{3}}+\lambda_{[2]}^{n_{3}} \lambda_{[11]}^{n_{1}}\right) \cdot[3] \chi_{22} \cdot A^{n_{2}}= \\
= & \frac{1}{\chi_{[1]}^{2}}\left\{H_{[1]}^{\left(n_{1}\right)} \bar{H}_{[1]}^{\left(n_{2}\right)} H_{[1]}^{\left(n_{3}\right)}+\chi_{[2]} \chi_{[11]}\left(\lambda_{[11]}^{n_{1}}-\lambda_{[2]}^{n_{1}}\right)\left(1-A^{n_{2}}\right)\left(\lambda_{[11]}^{n_{3}}-\lambda_{[2]}^{n_{3}}\right)\right\} \\
\chi_{[2]} H_{[2]}^{\left(n_{1}, \overline{n_{2}}, n_{3}\right)}= & \lambda_{[4]}^{n_{1}+n_{3}}\left\{\chi_{[4]}+\frac{\chi_{[21]} D_{3} D_{4}}{[4]^{2}}\left(D_{1} \cdot A^{n_{2}}+\frac{D_{0}^{2} D_{5}}{[2]^{2}[3]} \cdot(q A)^{2 n_{2}}\right)\right\}+ \\
& +\lambda_{[31]}^{n_{1}+n_{3}}\left\{\chi_{[31]}+\frac{[3] \chi_{[21]}}{[2][4]]^{2}}\left(D_{1} \cdot U_{[31]} \cdot A^{n_{2}}+\frac{\chi_{[1]}^{2} D_{3}}{[2]} \cdot V_{[31]} \cdot(q A)^{2 n_{2}}\right)\right\}+ \\
& +\lambda_{[22]}^{n_{1}+n_{3}} \cdot \chi_{[22]}\left\{1+\frac{D_{1} D_{-2}}{[2]} \cdot A^{n_{2}}+\frac{\chi_{[11]} D_{3} D_{-2}}{[2][3]} \cdot(q A)^{2 n_{2}}\right\}+ \\
& +\left(\lambda_{[4]}^{n_{1}} \lambda_{[31]}^{n_{3}}+\lambda_{[4]}^{n_{3}} \lambda_{[31]}^{n_{1}}\right) \frac{[2] \chi_{[21]} \chi_{[2]} D_{3}}{[4]^{2}}\left(A^{n_{2}}+\frac{D_{0} D_{4}}{[2]^{2}} \cdot(q A)^{2 n_{2}}\right)+ \\
& +\left(\lambda_{[31]}^{n_{1}} \lambda_{[22]}^{n_{3}}+\lambda_{[31]}^{n_{3}} \lambda_{[22]}^{n_{1}}\right) \cdot \chi_{[22]} \chi_{[2]}\left(A^{n_{2}}+\frac{D_{3} D_{-2}}{[4]} \cdot(q A)^{2 n_{2}}\right)+ \\
& +\left(\lambda_{[4]}^{n_{1}} \lambda_{[22]}^{n_{3}}+\lambda_{[4]}^{n_{3}} \lambda_{[22]}^{n_{1}}\right) \cdot \chi_{[4]} \chi_{[22]} \cdot(q A)^{2 n_{2}}
\end{aligned}
$$




$$
\begin{aligned}
& \chi_{[3]} H_{[3]}^{\left(n_{1}, \overline{n_{2}}, n_{3}\right)}= \\
& \lambda_{[6]}^{n_{1}+n_{3}}\left\{\chi_{[6]}+\frac{\chi_{[31]} D_{5} D_{6}}{[2][3]^{2}[4][5]^{2}[6]^{2}}\left(A^{n_{2}} \cdot[2][3]^{2}[4][5] D_{1} D_{4}+\right.\right. \\
& \left.\left.(q A)^{2 n_{2}} \cdot[3]^{2}[4] \chi_{[1]}^{2} D_{3} D_{7}+\left(q^{2} A\right)^{3 n_{2}} \cdot[2]^{2} \chi_{[2]}^{2} D_{7} D_{8}\right)\right\}+ \\
& \lambda_{[51]}^{n_{1}+n_{3}}\left\{\chi_{[51]}+\frac{\chi_{[31]}}{[2][3]^{2}[4][6]^{2}}\left(A^{n_{2}} \cdot[2][3]^{2} D_{1} D_{4} \cdot U_{[51]}+(q A)^{2 n_{2}} \cdot[3] \chi_{[1]}^{2} D_{3} D_{5} \cdot V_{[51]}+\right.\right. \\
& \left.\left.\left(q^{2} A\right)^{3 n_{2}} \cdot[2]^{2} \chi_{[2]}^{2} D_{5} D_{6} \cdot W_{[51]}\right)\right\}+ \\
& \lambda_{[42]}^{n_{1}+n_{3}}\left\{\chi_{[42]}+\frac{\chi_{[31]} \chi_{[1]}}{[2]^{2}[3][4][5]^{2}}\left(A^{n_{2}} \cdot[2][5] D_{1} \cdot U_{[42]}+(q A)^{2 n_{2}} \cdot[2] \chi_{[1]} D_{3} \cdot V_{[42]}+\right.\right. \\
& \left.\left.\left(q^{2} A\right)^{3 n_{2}} \cdot \chi_{[2]} D_{-2} D_{1} D_{5} \cdot W_{[42]}\right)\right\}+ \\
& \lambda_{[33]}^{n_{1}+n_{3}} \cdot \chi_{33}\left\{1+\frac{D_{1} D_{-2}}{[2]} \cdot A^{n_{2}}+\frac{\chi_{[11]} D_{3} D_{-2}}{[2][3]} \cdot(q A)^{2 n_{2}}+\frac{\chi_{[22]} D_{5} D_{-2}}{[3][4]} \cdot\left(q^{2} A\right)^{3 n_{2}}\right\}+ \\
& \left(\lambda_{[6]}^{n_{1}} \lambda_{[51]}^{n_{3}}+\lambda_{[51]}^{n_{3}} \lambda_{[6]}^{n_{1}}\right) \frac{[2] \chi_{[31]} \chi_{[2]} D_{5}}{[3][4][5][6]^{2}}\left(A^{n_{2}} \cdot[3][4] D_{4}+(q A)^{2 n_{2}} \cdot[3] \chi_{[1]} D_{3} D_{6}+\right. \\
& \left.\left(q^{2} A\right)^{3 n_{2}} \cdot \chi_{[3]} D_{6} D_{7}\right)+ \\
& \left(\lambda_{[6]}^{n_{1}} \lambda_{[42]}^{n_{3}}+\lambda_{[42]}^{n_{3}} \lambda_{[6]}^{n_{1}}\right) \frac{\chi_{[4]} \chi_{[31]} \chi_{[1]} D_{5}}{[2][5]^{2}[6]}\left((q A)^{2 n_{2}} \cdot[2][3]+\left(q^{2} A\right)^{3 n_{2}} D_{1} D_{6}\right)+ \\
& \left(\lambda_{[6]}^{n_{1}} \lambda_{[33]}^{n_{3}}+\lambda_{[33]}^{n_{3}} \lambda_{[6]}^{n_{1}}\right)\left(\left(q^{2} A\right)^{3 n_{2}} \frac{\chi_{[6]} \chi_{[31]} \chi_{[2]}}{[3]^{2}}\right)+ \\
& \left(\lambda_{[51]}^{n_{1}} \lambda_{[42]}^{n_{3}}+\lambda_{[42]}^{n_{3}} \lambda_{[51]}^{n_{1}}\right) \frac{\chi_{[31]} \chi_{[2]} \chi_{[1]}}{[2][3]^{2}[4][5][6]}\left(A^{n_{2}} \cdot[2]^{4}[6] D_{4}+(q A)^{2 n_{2}} \cdot[2][3] D_{3} \cdot V_{51 \mid 42}+\right. \\
& \left.\left(q^{2} A\right)^{3 n_{2}} \cdot[3] D_{1} D_{2} D_{5} \cdot W_{51 \mid 42}\right)+ \\
& \left(\lambda_{[51]}^{n_{1}} \lambda_{[33]}^{n_{3}}+\lambda_{[33]}^{n_{3}} \lambda_{[51]}^{n_{1}}\right) \frac{[5] \chi_{[41]} \chi_{[3]} \chi_{[2]}}{[3][4]^{2}[6]}\left((q A)^{2 n_{2}} \cdot[6]+\left(q^{2} A\right)^{3 n_{2}} \cdot D_{5} D_{-2}\right)+ \\
& \left(\lambda_{[42]}^{n_{1}} \lambda_{[33]}^{n_{3}}+\lambda_{[33]}^{n_{3}} \lambda_{[42]}^{n_{1}}\right) \frac{\chi_{[31]} \chi_{[2]}^{2}}{[2][3]^{2}[4][5]}\left(A^{n_{2}} \cdot[2][4][5]+(q A)^{2 n_{2}} \cdot[2][5] D_{3} D_{-2}+\right. \\
& \left.\left(q^{2} A\right)^{3 n_{2}} \cdot D_{5} D_{2} D_{-1} D_{-2}\right)
\end{aligned}
$$

Here

$$
\begin{aligned}
U_{[31]} & =[2] D_{3} D_{-2}+\left(D_{2}-D_{0}\right)^{2} \\
V_{[31]} & =D_{3} D_{-2}+D_{1} D_{0}-D_{2} D_{-1}=D_{3} D_{-2}+[2]=D_{1} D_{0}-[4] \\
U_{[51]} & =[4] D_{5} D_{-2}+\left(D_{4}-D_{0}\right)^{2} \\
V_{[51]} & =U_{[51]}-D_{2} D_{1}[2]\{q\}^{2}-D_{2}\left(D_{3}-D_{1}\right)[2] \\
W_{[51]} & =D_{5} D_{-2}+[4]+[2]=D_{5} D_{-2}+[2][3]=D_{2} D_{1}-[6] \\
U_{[42]} & =D_{3} D_{4}+[2]^{2} D_{1} D_{0}+D_{0} D_{-1}-[2]^{2}[3][4] \\
V_{[42]} & =[6] D_{2} D_{0}^{2}+D_{0}^{2} D_{-1}-[2]^{2}\left(\left(q^{7}+q^{3}+2 q+2 q^{-1}+q^{-3}+q^{-7}\right) D_{0}+D_{-5}\right)
\end{aligned}
$$




$$
\begin{aligned}
W_{[42]} & =[3] D_{2} D_{1}-[2][5] \\
V_{51 \mid 42} & =D_{5} D_{4}+[2] D_{2} D_{0}+D_{1} D_{0}-[2]^{3}[5] \\
W_{51 \mid 42} & =D_{2} D_{1}-[2][5]
\end{aligned}
$$

The reduced Jones polynomials $\left(A=q^{2}\right)$ :

$$
\begin{aligned}
& J_{[1]}=1+\frac{[3][4]}{[2]} q^{2\left(n_{1}+n_{2}+n_{3}\right)}+[3]\left(q^{2\left(n_{1}+n_{2}\right)}+q^{2\left(n_{1}+n_{3}\right)}+q^{2\left(n_{2}+n_{3}\right)}\right) \\
& J_{[2]}=1+\frac{[6]^{2}}{[4]^{2}} q^{2\left(n_{1}+n_{2}+n_{3}\right)}+\frac{[2][5][6][7]}{[3][4]^{2}} q^{6\left(n_{1}+n_{2}+n_{3}\right)}+ \\
& +\frac{[2][3][5][6]}{[4]^{2}}\left(q^{\left.6\left(n_{1}+n_{2}\right)+2 n_{3}\right)}+q^{\left.6\left(n_{1}+n_{3}\right)+2 n_{2}\right)}+q^{\left.6\left(n_{2}+n_{3}\right)+2 n_{1}\right)}\right)+ \\
& +\frac{[2]^{2}[3][5]}{[4]^{2}}\left(q^{6 n_{1}+2\left(n_{2}+n_{3}\right)}+q^{6 n_{2}+2\left(n_{1}+n_{3}\right)}+q^{6 n_{3}+2\left(n_{1}+n_{2}\right)}\right)+ \\
& +[5]\left(q^{6\left(n_{1}+n_{2}\right)}+q^{6\left(n_{1}+n_{3}\right)}+q^{6\left(n_{2}+n_{3}\right)}\right)+[3]\left(q^{2\left(n_{1}+n_{2}\right)}+q^{2\left(n_{1}+n_{3}\right)}+q^{2\left(n_{2}+n_{3}\right)}\right) \\
& J_{[3]}=1+\frac{[2][3][8]^{2}}{[4]^{3}[5]} q^{2\left(n_{1}+n_{2}+n_{3}\right)}+ \\
& +\frac{[2]^{3}[3]^{2}[5][7]}{[4][6]^{2}}\{q\}^{4} q^{6\left(n_{1}+n_{2}+n_{3}\right)}+\frac{[2][3][7][8][9][10]}{[4][5]^{2}[6]^{2}} q^{12\left(n_{1}+n_{2}+n_{3}\right)}+ \\
& +\frac{[2][3][7][8][9]}{[5][6]^{2}}\left(q^{12\left(n_{1}+n_{2}\right)+6 n_{3}}+q^{12\left(n_{1}+n_{3}\right)+6 n_{2}}+q^{12\left(n_{2}+n_{3}\right)+6 n_{1}}\right)+ \\
& +\frac{[3]^{2}[7][8]}{[5][6]}\left(q^{12\left(n_{1}+n_{2}\right)+2 n_{3}}+q^{12\left(n_{1}+n_{3}\right)+2 n_{2}}+q^{12\left(n_{2}+n_{3}\right)+2 n_{1}}\right)+ \\
& +\frac{[2]^{2}[3]^{2}[7][8]}{[4][6]^{2}}\left(q^{6\left(n_{1}+n_{2}\right)+12 n_{3}}+q^{6\left(n_{1}+n_{3}\right)+12 n_{2}}+q^{6\left(n_{2}+n_{3}\right)+12 n_{1}}\right)+ \\
& +\frac{[2]^{2}[3]^{2}[8]^{2}}{[4]^{3}[6]}\left(q^{6\left(n_{1}+n_{2}\right)+2 n_{3}}+q^{6\left(n_{1}+n_{3}\right)+2 n_{2}}+q^{6\left(n_{2}+n_{3}\right)+2 n_{1}}\right)+ \\
& +\frac{[2]^{4}[6]}{[4][5]}\left(q^{2\left(n_{1}+n_{2}\right)+6 n_{3}}+q^{2\left(n_{1}+n_{3}\right)+6 n_{2}}+q^{2\left(n_{2}+n_{3}\right)+6 n_{1}}\right)+ \\
& +[3]\left(q^{2\left(n_{1}+n_{2}\right)}+q^{2\left(n_{1}+n_{3}\right)}+q^{2\left(n_{2}+n_{3}\right)}\right)+ \\
& +[5]\left(q^{6\left(n_{1}+n_{2}\right)}+q^{6\left(n_{1}+n_{3}\right)}+q^{6\left(n_{2}+n_{3}\right)}\right)+ \\
& +[7]\left(q^{12\left(n_{1}+n_{2}\right)}+q^{12\left(n_{1}+n_{3}\right)}+q^{12\left(n_{2}+n_{3}\right)}\right)+ \\
& +\frac{[2][3]^{2}[7]}{[5][6]}\left(q^{12 n_{1}+6 n_{2}+2 n_{3}}+q^{12 n_{1}+6 n_{3}+2 n_{2}}+q^{12 n_{2}+6 n_{1}+2 n_{3}}+q^{12 n_{2}+6 n_{3}+2 n_{1}}\right. \\
& \left.+q^{12 n_{3}+6 n_{1}+2 n_{2}}+q^{12 n_{3}+6 n_{2}+2 n_{1}}\right)
\end{aligned}
$$

Genus $g=3$, the first symmetric representation [2].

$$
\begin{aligned}
& \chi_{[2]}^{2} H_{[2]}^{\left(n_{1}, n_{2}, n_{3}, n_{4}\right)}= \\
& \lambda_{[4]}^{n_{1}+n_{2}+n_{3}+n_{4}} \cdot\left(\frac{\chi_{[4]}^{4}}{\chi_{[2]}^{2}}+\frac{[2]}{[3]^{2}[4]^{3}} \chi_{[4]} \chi_{[21]}\left(\frac{[3]\left\{A q^{4}\right\}^{2}\{A\}+\left\{A q^{3}\right\}^{2}\left\{A q^{2}\right\}}{\{q\}^{3}}+\frac{[3][4]\left\{A q^{3}\right\}}{\{q\}}\right)\right)+
\end{aligned}
$$




$$
\begin{aligned}
& \left(\lambda_{[4]}^{n_{1}+n_{2}+n_{3}} \lambda_{[22]}^{n_{4}}+\text { perms }\right)\left(\frac{\chi_{[4]}^{3} \chi_{[22]}}{\chi_{[2]}^{2}}-\frac{[2]^{2}}{[3]^{2}[4]^{2}} \chi_{[4]} \chi_{[22]} \frac{\left\{A q^{3}\right\}^{2}+\left\{A q^{4}\right\}\{A\}}{\{q\}^{2}}\right)+ \\
& \left(\lambda_{[4]}^{n_{1}+n_{2}} \lambda_{[22]}^{n_{3}+n_{4}}+\text { perms }\right)\left(\frac{\chi_{[4]}^{2} \chi_{[22]}^{2}}{\chi_{[2]}^{2}}+\frac{\chi_{[4]} \chi_{[32]} \chi_{[1]}}{[3]^{2}}\right)+ \\
& \left(\lambda_{[4]}^{n_{1}} \lambda_{[22]}^{n_{2}+n_{3}+n_{4}}+\text { perms }\right)\left(\frac{\chi_{[4]} \chi_{[22]}^{3}}{\chi_{[2]}^{2}}-\frac{\chi_{[4]} \chi_{[22]} \chi_{[11]}}{[3]^{2}}\right)+ \\
& \left(\lambda_{[22]}^{n_{1}+n_{2}+n_{3}+n_{4}}+\text { perms }\right)\left(\frac{\chi_{[22]}^{4}}{\chi_{[2]}^{2}}+\frac{\chi_{[22]}\left(\chi_{[4]}+\chi_{[31]}\right)}{[2]^{2}[3]^{2}}\left(\chi_{[11]}+[3]\right)\right)+ \\
& \left(\lambda_{[4]}^{n_{1}+n_{2}+n_{3}} \lambda_{[31]}^{n_{4}}+\text { perms }\right)\left(\frac{\chi_{[4]}^{3} \chi_{[31]}}{\chi_{[2]}^{2}}-\frac{[2]}{[3][4]^{3}} \chi_{[4]} \chi_{[21]}\left([2] \frac{q^{3} A^{2}-q^{-3} A^{-2}}{q-q^{-1}} \frac{\left\{q^{3} A\right\}}{\{q\}}+[3] \chi_{[1]}\right)\right)+ \\
& \left(\lambda_{[4]}^{n_{1}+n_{2}} \lambda_{[31]}^{n_{3}+n_{4}}+\text { perms }\right)\left(\frac{\chi_{[4]}^{2} \chi_{[31]}^{2}}{\chi_{[2]}^{2}}+\frac{[2]}{[4]^{3}} \chi_{[4]} \chi_{[21]}\left(\frac{q^{4} A^{3}-q^{-4} A^{-3}}{q-q^{-1}}+[2] \frac{\{q A\}}{\{q\}}\right)\right)+ \\
& \left(\lambda_{[4]}^{n_{1}} \lambda_{[31]}^{n_{2}+n_{3}+n_{4}}+\text { perms }\right)\left(\frac{\chi_{[4]} \chi_{[31]}^{3}}{\chi_{[2]}^{2}}-\frac{[3]}{[4]^{3}} \chi_{[4]} \chi_{[21]}\left(\left(q^{2} A^{3}+q^{-2} A^{-3}\right)+2 \frac{\{q A\}}{\{q\}}\right)\right)+(\text { A. } 11) \\
& \lambda_{[31]}^{n_{1}+n_{2}+n_{3}+n_{4}}\left(\frac{\chi_{[31]}^{4}}{\chi_{[2]}^{2}}+\frac{1}{[4]^{3}} \chi_{[31]} \chi_{[2]}\left(\left(q^{3} A^{4}+\frac{1}{q^{3} A^{4}}\right)+\right.\right. \\
& \left.\frac{\left(4 q^{3}-3 q+\frac{2}{q}+\frac{1}{q^{3}}\right) A^{2}-2[4]+\left(\frac{4}{q^{3}}-\frac{3}{q}+2 q+q^{3}\right) \frac{1}{A^{2}}}{\left(q-q^{-1}\right)^{2}}\right)+ \\
& \left(\lambda_{[31]}^{n_{1}+n_{2}+n 3} \lambda_{[22]}^{n_{4}}+\text { perms }\right)\left(\frac{\chi_{[22]} \chi_{[31]}^{3}}{\chi_{[2]}^{2}}+\frac{[3]}{[4]^{3}} \chi_{[22]} \chi_{[3]}\left(\left(q^{2} A^{3}+q^{-2} A^{-3}\right)-\right.\right. \\
& \left.\left.2 \frac{\{A / q\}}{\{q\}}-\left(q^{3}+q^{-3}\right) \chi_{[1]}\right)\right)+ \\
& \left(\lambda_{[31]}^{n_{1}+n_{2}} \lambda_{[22]}^{n_{3}+n_{4}}+\text { perms }\right)\left(\frac{\chi_{[22]}^{2} \chi_{[31]}^{2}}{\chi_{[2]}^{2}}+\frac{1}{[2][4]^{2}} \chi_{[22]} \chi_{[3]} \chi_{[1]}\left(\left(q A^{2}+q^{-1} A^{-2}\right)+[2][3]\right)\right)+ \\
& \left(\lambda_{[31]}^{n_{1}} \lambda_{[22]}^{n_{2}+n_{3}+n_{4}}+\text { perms }\right)\left(\frac{\chi_{[22]}^{3} \chi_{[31]}}{\chi_{[2]}^{2}}+\frac{1}{[2]^{2}[3][4]} \chi_{22]} \chi_{[3]} \chi_{[1]}\left(\left(A+A^{-1}\right) \chi_{[1]}-[2][3]\right)\right)+ \\
& \left(\lambda_{[4]}^{n_{1}+n_{2}} \lambda_{[31]}^{n_{3}} \lambda_{[22]}^{n_{4}}+\text { perms }\right)\left(\frac{\chi_{[4]}^{2} \chi_{[31]} \chi_{[22]}}{\chi_{[2]}^{2}}+\frac{[2]^{2}}{[3][4]^{2}} \chi_{[4]} \chi_{[22]} \frac{q^{3} A^{2}-q^{-3} A^{-2}}{q-q^{-1}}\right)+ \\
& \left(\lambda_{[4]}^{n_{1}} \lambda_{[31]}^{n_{2}+n_{3}} \lambda_{[22]}^{n_{4}}+\text { perms }\right)\left(\frac{\chi_{[4]} \chi_{[31]}^{2} \chi_{[22]}}{\chi_{[2]}^{2}}-\frac{[2]}{[4]^{2}} \chi_{[4]} \chi_{[22]}\left(q A^{2}+q^{-1} A^{-2}\right)\right)+ \\
& \left(\lambda_{[4]}^{n_{1}} \lambda_{[31]}^{n_{2}} \lambda_{[22]}^{n_{3}+n_{4}}+\text { perms }\right)\left(\frac{\chi_{[4]} \chi_{[31]} \chi_{[22]}^{2}}{\chi_{[2]}^{2}}-\frac{1}{[3][4]} \chi_{[4]} \chi_{[22]} \chi_{[1]}\left(A+A^{-1}\right)\right)
\end{aligned}
$$


The result (3.1) of the present paper is that this long and strangely-looking expression is nothing else but

$$
\begin{aligned}
& H_{[2]}^{\left(n_{1}, n_{2}, n_{3}, n_{4}\right)}= \\
& \sum_{k=0}^{3} \bar{\Delta}_{k} \cdot \prod_{i=1}^{4}\left(\sum_{m=0}^{3} a_{k m} \lambda_{m}^{n_{i}}\right) \stackrel{(B .1)}{=} \prod_{i=1}^{4}\left(\frac{D_{0} D_{-1}}{[2][3]}+\frac{D_{2} D_{-1}}{[4]} \cdot\left(-q^{2}\right)^{n_{i}}+\frac{D_{3} D_{2}}{[3][4]} \cdot q^{6 n_{i}}\right)+ \\
& \quad+D_{1} D_{-1} \cdot \prod_{i=1}^{4}\left(\frac{D_{0}}{[2][3]}+\frac{D_{2}-D_{0}}{[4]} \cdot\left(-q^{2}\right)^{n_{i}}-\frac{[2] D_{3}}{[3][4]} \cdot q^{6 n_{i}}\right) \\
& \quad+\frac{D_{3} D_{0}^{2} D_{-1}}{[2]^{2}} \cdot \prod_{i=1}^{4}\left(\frac{1}{[3]}-\frac{[2]}{[4]} \cdot\left(-q^{2}\right)^{n_{i}}+\frac{[2]}{[3][4]} \cdot q^{6 n_{i}}\right)
\end{aligned}
$$

which is not only shorter, but also a much better structured expression, moreover, generalizable to arbitrary genus and representation.

\section{B List of coefficients $a_{k m}, \bar{a}_{k m}$ and $\overline{\bar{a}}_{k m}$}

We list in this appendix both the coefficients of all three matrices $\mathcal{A}, \overline{\mathcal{A}}$ and $\overline{\overline{\mathcal{A}}}$ and the two Racah matrices corresponding to $\mathcal{A}, \overline{\mathcal{A}}$, since the third Racah matrix is obtained from that for $\mathcal{A}$ just by transposing.

Coefficients $\boldsymbol{a}_{\boldsymbol{k m}}$. Coefficients $a_{k m}$ entering contributions of the parallel braid:

$$
\begin{aligned}
& \mathcal{A}_{[1]}=\frac{1}{\chi_{[1]}}\left(\begin{array}{cc}
\chi_{[11]} & \chi_{[2]} \\
\frac{\chi_{[1]}}{[2]} & -\frac{\chi_{[1]}}{[2]}
\end{array}\right)=\frac{1}{[2]}\left(\begin{array}{cc}
D_{-1} & D_{1} \\
1 & -1
\end{array}\right) \\
& \mathcal{A}_{[2]}=\frac{1}{\chi_{[2]}}\left(\begin{array}{ccc}
\chi_{[22]} & \chi_{[31]} & \chi_{[4]} \\
\frac{\chi_{[2]} \cdot D_{0}}{[2][3]} \frac{\chi_{[2]} \cdot\left(D_{2}-D_{0}\right)}{[4]} & -\frac{[2] \chi_{[2]} \cdot D_{3}}{[3][4]} \\
\frac{\chi_{[2]}}{[3]} & -\frac{[2] \chi_{[2]} \cdot}{[4]} & \frac{[2] \chi_{[2]}}{[3][4]}
\end{array}\right)=\frac{1}{[3]}\left(\begin{array}{ccc}
\frac{1}{[2]} D_{0} D_{-1} & \frac{[3]}{[4]} D_{2} D_{-1} & \frac{1}{[4]} D_{3} D_{2} \\
\frac{1}{[2]} D_{0} & \frac{[3]}{[4]}\left(D_{2}-D_{0}\right) & -\frac{[2]}{[4]} D_{3} \\
1 & -\frac{[2][3]}{[4]} & \frac{[2]}{[4]}
\end{array}\right) \\
& \mathcal{A}_{[3]}=\frac{1}{[4]}\left(\begin{array}{cccc}
\frac{1}{[2][3]} D_{1} D_{0} D_{-1} & \frac{[3]}{[2][5]} D_{3} D_{0} D_{-1} & \frac{1}{[6]} D_{4} D_{3} D_{-1} & \frac{1}{[5][6]} D_{5} D_{4} D_{3} \\
\frac{1}{[2][3]} D_{1} D_{0} & \frac{1}{[2][5]}\left([2] D_{4}-D_{-1}\right) D_{0} & \frac{1}{[3][6]} D_{4}\left(D_{5}-[2]^{2} D_{-1}\right) & -\frac{[3]}{[5][6]} D_{5} D_{4} \\
\frac{1}{[3]} D_{1} & \frac{1}{[5]}\left(D_{5}-[2] D_{0}\right) & \frac{[2]}{[3][6]}\left(-[2]^{2} D_{5}+D_{-1}\right) & \frac{[2][3]}{[5][6]} D_{5} \\
1 & -\frac{[3]^{2}}{[5]} & \frac{[2][3]}{[6]} & -\frac{[2][3]}{[5][6]}
\end{array}\right)
\end{aligned}
$$




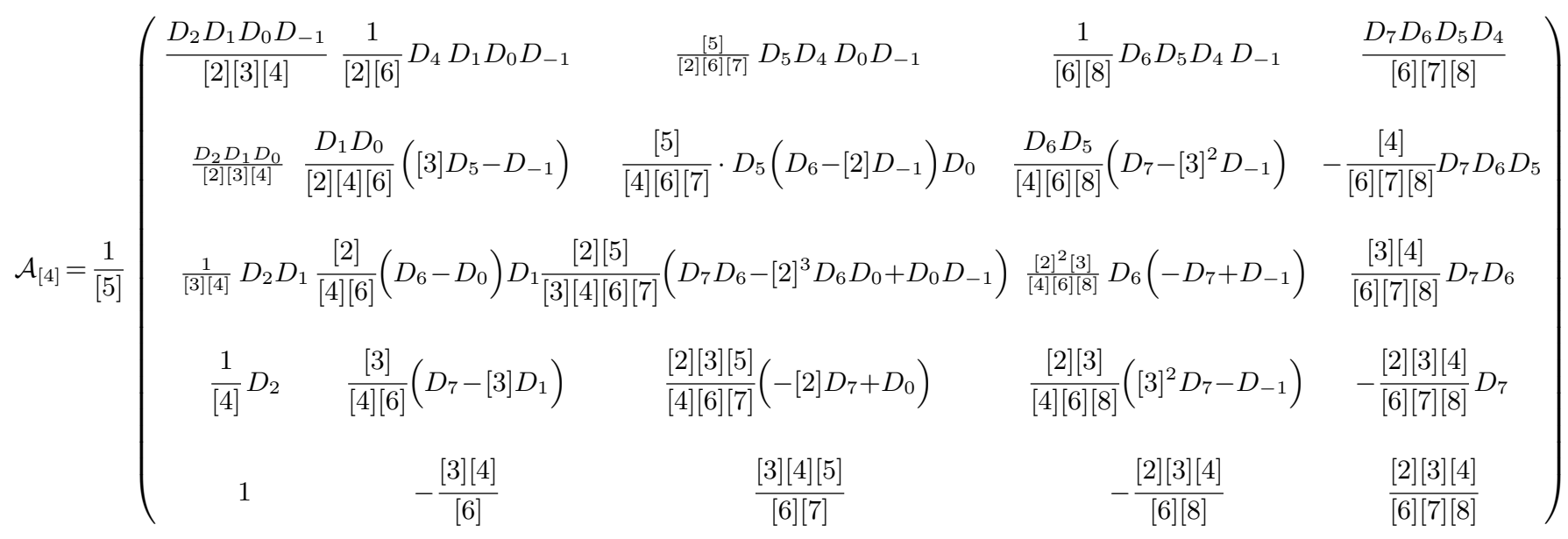

The Racah matrix associated with $a_{k m}$ are

$$
\begin{gathered}
S_{[1]}=\frac{1}{\sqrt{[2] D_{0}}}\left(\begin{array}{cc}
\sqrt{D_{-1}} & \sqrt{D_{1}} \\
\sqrt{D_{1}} & -\sqrt{D_{-1}}
\end{array}\right) \\
S_{[2]}=\frac{1}{[3]}\left(\begin{array}{ccc}
\sqrt{\frac{1}{\Delta_{0}}} \cdot \frac{1}{[2]} D_{0} D_{-1} & \sqrt{\frac{1}{\Delta_{1}}} \cdot \frac{[3]}{[4]} D_{2} D_{-1} & \sqrt{\frac{1}{\Delta_{2}}} \cdot \frac{1}{[4]} D_{3} D_{2} \\
\sqrt{\frac{\bar{\Delta}_{1}}{\Delta_{0}}} \cdot \frac{1}{[2]} D_{0} & \sqrt{\frac{\bar{\Delta}_{1}}{\Delta_{1}}} \cdot \frac{[3]}{[4]}\left(D_{2}-D_{0}\right) & -\sqrt{\frac{\bar{\Delta}_{1}}{\Delta_{2}}} \cdot \frac{[2]}{[4]} D_{3} \\
\sqrt{\frac{\bar{\Delta}_{2}}{\Delta_{0}}} & -\sqrt{\frac{\bar{\Delta}_{2}}{\Delta_{1}}} \cdot \frac{[2][3]}{[4]} & \sqrt{\frac{\bar{\Delta}_{2}}{\Delta_{2}}} \cdot \frac{[2]}{[4]}
\end{array}\right)
\end{gathered}
$$

Coefficients $\overline{\boldsymbol{a}}_{\boldsymbol{k m}}$. Coefficients $\bar{a}_{k m}$ entering contributions of the antiparallel braid with even crossings:

$$
\begin{aligned}
& \overline{\mathcal{A}}_{1}=\frac{1}{D_{0}}\left(\begin{array}{cc}
1 & D_{1} D_{-1} \\
1 & -1
\end{array}\right)=\frac{1}{\chi_{[1]}}\left(\begin{array}{cc}
1 & \bar{\Delta}_{1} \\
1 & -1
\end{array}\right), \\
& \overline{\mathcal{A}}_{2}=\frac{1}{\chi_{[2]}}\left(\begin{array}{ccc}
1 & \bar{\Delta}_{1} & \bar{\Delta}_{2} \\
1 \frac{D_{1}}{[2] D_{2}}\left(D_{3} D_{-1}-1\right) & -\frac{D_{0}^{2} D_{3}}{[2] D_{2}} \\
1 & -[2] \frac{D_{1}}{D_{2}} & \frac{D_{0}}{D_{2}}
\end{array}\right),
\end{aligned}
$$




$$
\overline{\mathcal{A}}_{3}=\frac{1}{\chi_{[3]}}\left(\begin{array}{cccc}
1 & \bar{\Delta}_{1} & \bar{\Delta}_{2} & \bar{\Delta}_{3} \\
1 \frac{D_{1}}{[3] D_{3}}\left([2] D_{3} D_{0}-[3]^{2}\right) & \frac{D_{0}^{2}}{[2]^{2}[3]}\left(D_{4} D_{0}-[3]^{2}\right) & -\frac{D_{5} D_{1}^{2} D_{0}^{2}}{[2]^{2}[3] D_{3}} \\
1 & \frac{D_{1}}{[3] D_{3}}\left(D_{4} D_{0}-[3]^{2}\right) & -\frac{D_{0}}{[3] D_{4}}\left([2] D_{4} D_{1}-[3]^{2}\right) & \frac{D_{5} D_{1}^{2} D_{0}}{[3] D_{4} D_{3}} \\
1 & -\frac{[3] D_{1}}{D_{3}} & \frac{[3] D_{0}}{D_{4}} & -\frac{D_{1} D_{0}}{D_{4} D_{3}}
\end{array}\right),
$$

$$
\begin{aligned}
& \left(\begin{array}{ll}
1 & \bar{\Delta}_{1}
\end{array}\right. \\
& \bar{\Delta}_{2} \\
& \bar{\Delta}_{3}
\end{aligned}
$$

The Racah matrix associated with $\bar{a}_{k m}$ are

$$
\begin{aligned}
& \bar{S}_{1}=\frac{1}{\chi_{[1]}}\left(\begin{array}{cc}
1 & \sqrt{\bar{\Delta}_{1}} \\
\sqrt{\bar{\Delta}_{1}} & -1
\end{array}\right), \\
& \bar{S}_{2}=\frac{1}{\chi_{[2]}}\left(\begin{array}{ccc}
1 & \sqrt{\bar{\Delta}_{1}} & \sqrt{\bar{\Delta}_{2}} \\
\sqrt{\bar{\Delta}_{1}} & \frac{D_{1}}{[2] D_{2}}\left(D_{3} D_{-1}-1\right) & -\frac{D_{0}}{D_{2}} \sqrt{D_{3} D_{1}} \\
\sqrt{\bar{\Delta}_{2}} & -\frac{D_{0}}{D_{2}} \sqrt{D_{3} D_{1}} & \frac{D_{0}}{D_{2}}
\end{array}\right)
\end{aligned}
$$

Coefficients $\overline{\overline{\boldsymbol{a}}}_{\boldsymbol{k} \boldsymbol{m}}$. Coefficients $\overline{\bar{a}}_{k m}$ entering contributions of the antiparallel braid with odd crossings:

$$
\begin{aligned}
& \overline{\mathcal{A}}_{[1]}=\frac{1}{D_{0}}\left(\begin{array}{cc}
1 & D_{1} \\
1 & -D_{-1}
\end{array}\right) \\
& \overline{\overline{\mathcal{A}}}_{[2]}=\frac{1}{[3]}\left(\begin{array}{ccc}
\frac{1}{[2] \chi_{[22]}} D_{0} D_{-1} & \frac{\Delta_{1}}{[2] \chi_{[22]}} D_{0} & \frac{\Delta_{2}}{\chi_{[22]}} \\
\frac{[3]}{[4] \chi_{[31]}} D_{2} D_{-1} & \frac{[3] \Delta_{1}}{[4] \chi_{[31]}}\left(D_{2}-D_{0}\right) & -\frac{[2][3] \Delta_{2}}{[4] \chi_{[31]}} \\
\frac{1}{[4] \chi_{[4]}} D_{3} D_{2} & -\frac{[2] \Delta_{1}}{[4] \chi_{[4]}} D_{3} & \frac{[2] \Delta_{2}}{[4] \chi_{[4]}}
\end{array}\right)=\frac{1}{D_{0} D_{1} D_{2}}\left(\begin{array}{ccc}
{[2] D_{2}} & {[2] D_{2} D_{1}} & D_{3} D_{2} D_{0} \\
{[2] D_{2}} & {[2] D_{1}\left(D_{2}-D_{0}\right)} & -D_{3} D_{0}^{2} \\
{[2] D_{2}} & -[2]^{2} D_{1} D_{-1} & D_{0}^{2} D_{-1}
\end{array}\right)
\end{aligned}
$$




$$
\begin{aligned}
& \overline{\overline{\mathcal{A}}}_{[3]}=\frac{1}{[4]}\left(\begin{array}{cccc}
\frac{1}{[2][3] \chi_{[33]}} D_{1} D_{0} D_{-1} & \frac{\Delta_{1}}{[2][3] \chi_{[33]}} D_{1} D_{0} & \frac{\Delta_{2}}{[3] \chi_{[33]}} D_{1} & \frac{\Delta_{3}}{\chi_{[33]}} \\
\frac{[3]}{[2][5] \chi_{[42]}} D_{3} D_{0} D_{-1} & \frac{\Delta_{1}}{[2][5] \chi_{[42]}}\left([2] D_{4}-D_{-1}\right) D_{0} & \frac{\Delta_{2}}{[5] \chi_{[42]}}\left(D_{5}-[2] D_{0}\right) & -\frac{[3]^{2} \Delta_{3}}{[5] \chi_{[42]}} \\
\frac{1}{[6] \chi_{[51]}} D_{4} D_{3} D_{-1} & \frac{\Delta_{1}}{[3][6] \chi_{[51]}} D_{4}\left(D_{5}-[2]^{2} D_{-1}\right) & \frac{[2] \Delta_{2}}{[3][6] \chi_{[51]}}\left(-[2]^{2} D_{5}+D_{-1}\right) & \frac{[2][3] \Delta_{3}}{[6] \chi_{[51]}} \\
\frac{1}{[5][6] \chi_{[6]}} D_{5} D_{4} D_{3} & -\frac{[3] \Delta_{1}}{[5][6] \chi_{[6]}} D_{5} D_{4} & \frac{[2][3] \Delta_{2}}{[5][6] \chi_{[6]}} D_{5} & -\frac{[2][3] \Delta_{3}}{[5][6] \chi_{[6]}}
\end{array}\right)= \\
& =\frac{1}{D_{4} D_{3} D_{2} D_{1} D_{0}}\left(\begin{array}{cccc}
{[2][3] D_{4} D_{3}} & {[2][3] D_{4} D_{3} D_{1}} & {[3] D_{4} D_{3}^{2} D_{0}} & D_{5} D_{4} D_{3} D_{1} D_{0} \\
{[2][3] D_{3} D_{4}} & {[2] D_{4} D_{1}\left([2] D_{4}-D_{-1}\right)} & D_{4} D_{3} D_{0}\left(D_{5}-[2] D_{0}\right) & -D_{5} D_{4} D_{1}^{2} D_{0} \\
{[2][3] D_{4} D_{3}} & {[2] D_{4} D_{1}\left(D_{5}-[2]^{2} D_{-1}\right)} & D_{3} D_{0}^{2}\left(-[2]^{2} D_{5}+D_{-1}\right) & D_{5} D_{1}^{2} D_{0}^{2} \\
{[2][3] D_{4} D_{3}} & -[2][3]^{2} D_{4} D_{1} D_{-1} & {[3]^{2} D_{3} D_{0}^{2} D_{-1}} & -D_{1}^{2} D_{0}^{2} D_{-1}
\end{array}\right)
\end{aligned}
$$

Open Access. This article is distributed under the terms of the Creative Commons Attribution License (CC-BY 4.0), which permits any use, distribution and reproduction in any medium, provided the original author(s) and source are credited.

\section{References}

[1] N.Y. Reshetikhin and V.G. Turaev, Ribbon graphs and their invariants derived from quantum groups, Commun. Math. Phys. 127 (1990) 1 [INSPIRE].

[2] E. Guadagnini, M. Martellini and M.Mintchev, Chern-Simons field theory and quantum groups, Springer, Lect. Notes. Phys. 370 (1990) 307.

[3] E. Guadagnini, M. Martellini and M. Mintchev, Chern-Simons Holonomies and the Appearance of Quantum Groups, Phys. Lett. B 235 (1990) 275 [InSPIRE].

[4] A. Morozov and A. Smirnov, Chern-Simons Theory in the Temporal Gauge and Knot Invariants through the Universal Quantum R-Matrix, Nucl. Phys. B 835 (2010) 284 [arXiv: 1001.2003] [INSPIRE].

[5] A. Smirnov, Notes on Chern-Simons Theory in the Temporal Gauge, arXiv:0910.5011 [INSPIRE].

[6] A. Mironov, A. Morozov and A. Morozov, Character expansion for HOMFLY polynomials. I. Integrability and difference equations, arXiv:1112.5754 [INSPIRE].

[7] H. Itoyama, A. Mironov, A. Morozov and A. Morozov, Character expansion for HOMFLY polynomials. III. All 3-Strand braids in the first symmetric representation, Int. J. Mod. Phys. A 27 (2012) 1250099 [arXiv: 1204.4785] [INSPIRE].

[8] A. Anokhina, A. Mironov, A. Morozov and A. Morozov, Racah coefficients and extended HOMFLY polynomials for all 5-, 6- and 7-strand braids, Nucl. Phys. B 868 (2013) 271 [arXiv:1207.0279] [INSPIRE]. 
[9] H. Itoyama, A. Mironov, A. Morozov and A. Morozov, Eigenvalue hypothesis for Racah matrices and HOMFLY polynomials for 3-strand knots in any symmetric and antisymmetric representations, Int. J. Mod. Phys. A 28 (2013) 1340009 [arXiv:1209.6304] [INSPIRE].

[10] A. Anokhina, A. Mironov, A. Morozov and A. Morozov, Colored HOMFLY polynomials as multiple sums over paths or standard Young tableaux, Adv. High Energy Phys. 2013 (2013) 931830 [arXiv: 1304.1486] [INSPIRE].

[11] A. Anokhina and A. Morozov, Cabling procedure for the colored HOMFLY polynomials, Teor. Mat. Fiz. 178 (2014) 3 [arXiv:1307.2216] [INSPIRE].

[12] A. Mironov, A. Morozov and A. Morozov, Character expansion for HOMFLY polynomials. II. Fundamental representation. Up to five strands in braid, JHEP 03 (2012) 034 [arXiv: 1112.2654] [INSPIRE].

[13] R.K. Kaul and T.R. Govindarajan, Three-dimensional Chern-Simons theory as a theory of knots and links, Nucl. Phys. B 380 (1992) 293 [hep-th/9111063] [INSPIRE].

[14] P. Rama Devi, T.R. Govindarajan and R.K. Kaul, Three-dimensional Chern-Simons theory as a theory of knots and links. 3. Compact semisimple group, Nucl. Phys. B 402 (1993) 548 [hep-th/9212110] [INSPIRE].

[15] P. Ramadevi, T.R. Govindarajan and R.K. Kaul, Knot invariants from rational conformal field theories, Nucl. Phys. B 422 (1994) 291 [hep-th/9312215] [INSPIRE].

[16] P. Ramadevi and T. Sarkar, On link invariants and topological string amplitudes, Nucl. Phys. B 600 (2001) 487 [hep-th/0009188] [InSPIRE].

[17] Zodinmawia and P. Ramadevi, $\mathrm{SU}(N)$ quantum Racah coefficients 83 non-torus links, Nucl. Phys. B 870 (2013) 205 [arXiv:1107.3918] [InSPIRE].

[18] Zodinmawia and P. Ramadevi, Reformulated invariants for non-torus knots and links, arXiv:1209.1346 [INSPIRE].

[19] J.W. Alexander, Topological invariants of knots and links, Trans. Am. Math. Soc. 30 (1928) 275 .

[20] J.H. Conway, An Enumeration of Knots and Links, and Some of Their Algebraic Properties, in Computational Problems in Abstract Algebra, J. Leech ed., Oxford, U.K. (1967), Pergamon Press, (1970), pg. 329-358.

[21] V.F.R. Jones, Index for subfactors, Invent. Math. 72 (1983) 1.

[22] V.F.R. Jones, A polynomial invariant for links via von Neumann algebras, Bull. Am. Math. Soc. 12 (1985) 103.

[23] V.F.R. Jones, Hecke algebra representations of braid groups and link polynomials, Annals Math. 126 (1987) 335 [INSPIRE].

[24] L. Kauffman, State models and the Jones polynomial, Topology 26 (1987) 395.

[25] P. Freyd, D. Yetter, J. Hoste, W.B.R. Lickorish, K. Millet and A. Ocneanu, A new polynomial invariant of knots and links, Bull. Am. Math. Soc. 12 (1985) 239.

[26] J.H. Przytycki and K.P. Traczyk, Invariants of links Conway type, Kobe J. Math. 4 (1988) 115.

[27] E. Witten, Quantum Field Theory and the Jones Polynomial, Commun. Math. Phys. 121 (1989) 351 [INSPIRE]. 
[28] A. Mironov and A. Morozov, Equations on knot polynomials and 3d/5d duality, AIP Conf. Proc. 1483 (2012) 189 [arXiv:1208.2282] [INSPIRE].

[29] M.Rosso and V.F.R. Jones, On the invariants of torus knots derived from quantum groups, J. Knot Theory Ramifications 2 (1993) 97.

[30] X.-S. Lin and H. Zheng, On the Hecke algebras and the colored HOMFLY polynomial Trans. Am. Math. Soc. 362 (2010) 1 [math/0601267].

[31] N.M. Dunfield, S. Gukov and J. Rasmussen, The Superpolynomial for knot homologies, math/0505662 [INSPIRE].

[32] A. Mironov, A. Morozov and S. Natanzon, Complete Set of Cut-and-Join Operators in Hurwitz-Kontsevich Theory, Theor. Math. Phys. 166 (2011) 1 [arXiv:0904.4227] [InSPIRE].

[33] A. Mironov, A. Morozov and S. Natanzon, Algebra of differential operators associated with Young diagrams, J. Geom. Phys. 62 (2012) 148 [arXiv:1012.0433] [InSPIRE].

[34] M. Tierz, Soft matrix models and Chern-Simons partition functions, Mod. Phys. Lett. A 19 (2004) 1365 [hep-th/0212128] [INSPIRE].

[35] A. Brini, B. Eynard and M. Mariño, Torus knots and mirror symmetry, Annales Henri Poincaré 13 (2012) 1873 [arXiv:1105.2012] [INSPIRE].

[36] M. Aganagic and S. Shakirov, Knot Homology and Refined Chern-Simons Index, Commun. Math. Phys. 333 (2015) 187 [arXiv:1105.5117] [INSPIRE].

[37] P. Dunin-Barkowski, A. Mironov, A. Morozov, A. Sleptsov and A. Smirnov, Superpolynomials for toric knots from evolution induced by cut-and-join operators, JHEP 03 (2013) 021 [arXiv:1106.4305] [INSPIRE].

[38] I. Cherednik, Jones polynomials of torus knots via DAHA, arXiv:1111.6195 [INSPIRE].

[39] A. Mironov, A. Morozov and A. Morozov, Evolution method and "differential hierarchy" of colored knot polynomials, AIP Conf. Proc. 1562 (2013) 123 [arXiv:1306.3197] [INSPIRE].

[40] H. Itoyama, A. Mironov, A. Morozov and A. Morozov, HOMFLY and superpolynomials for figure eight knot in all symmetric and antisymmetric representations, JHEP 07 (2012) 131 [arXiv: 1203.5978] [INSPIRE].

[41] S. Arthamonov, A. Mironov and A. Morozov, Differential hierarchy and additional grading of knot polynomials, Theor. Math. Phys. 179 (2014) 509 [arXiv:1306.5682] [InSPIRE].

[42] S. Gukov and M. Stosic, Homological Algebra of Knots and BPS States, Proc. Symp. Pure Math. 85 (2012) 125 [arXiv:1112.0030] [INSPIRE].

[43] H. Fuji, S. Gukov and P. Sulkowski, Super-A-polynomial for knots and BPS states, Nucl. Phys. B 867 (2013) 506 [arXiv: 1205.1515] [INSPIRE].

[44] H. Fuji, S. Gukov, M. Stosic and P. Sulkowski, 3d analogs of Argyres-Douglas theories and knot homologies, JHEP 01 (2013) 175 [arXiv:1209.1416] [INSPIRE].

[45] S. Nawata, P. Ramadevi and Zodinmawia, Colored Kauffman Homology and Super-A-polynomials, JHEP 01 (2014) 126 [arXiv:1310.2240] [INSPIRE].

[46] S. Nawata, P. Ramadevi, Zodinmawia and X. Sun, Super-A-polynomials for Twist Knots, JHEP 11 (2012) 157 [arXiv: 1209.1409] [INSPIRE].

[47] H. Fuji and P. Sulkowski, Super-A-polynomial, arXiv:1303.3709 [INSPIRE]. 
[48] A. Morozov, Special colored Superpolynomials and their representation-dependence, JHEP 12 (2012) 116 [arXiv: 1208.3544] [INSPIRE].

[49] A. Morozov, The first-order deviation of superpolynomial in an arbitrary representation from the special polynomial, JETP Lett. 97 (2013) 171 [arXiv:1211.4596] [INSPIRE].

[50] E. Gorsky, S. Gukov and M. Stosic, Quadruply-graded colored homology of knots, arXiv:1304.3481 [INSPIRE].

[51] S. Arthamonov, A. Mironov, A. Morozov and A. Morozov, Link polynomial calculus and the AENV conjecture, JHEP 04 (2014) 156 [arXiv: 1309.7984] [INSPIRE].

[52] A. Alexandrov, A. Mironov, A. Morozov and A. Morozov, Towards matrix model representation of HOMFLY polynomials, JETP Lett. 100 (2014) 271 [arXiv:1407.3754] [INSPIRE].

[53] A. Alexandrov and D. Melnikov, Matrix integral expansion of colored Jones polynomials for figure-eight knot, JETP Lett. 101 (2015) 51 [arXiv:1411.5698] [INSPIRE].

[54] A. Anokhina, A. Mironov, A. Morozov and A. Morozov, Knot polynomials in the first non-symmetric representation, Nucl. Phys. B 882 (2014) 171 [arXiv:1211.6375] [InSPIRE].

[55] J. Gu and H. Jockers, A note on colored HOMFLY polynomials for hyperbolic knots from WZW models, Commun. Math. Phys. 338 (2015) 393 [arXiv:1407.5643] [InSPIRE].

[56] A. Mironov, A. Morozov and A. Morozov, On colored HOMFLY polynomials for twist knots, Mod. Phys. Lett. A 29 (2014) 1450183 [arXiv:1408.3076] [InSPIRE].

[57] A. Kawauchi, Survey on knot theory, Springer, (1996).

[58] H. Morton and P. Cromwell, Distinguishing mutants by knot polynomials, J. Knot Theory Ramifications 5 (1996) 225.

[59] P.R. Cromwell, Knots and links, Cambridge University Press, (2004).

[60] D. Galakhov, D. Melnikov, A. Mironov, A. Morozov and A. Sleptsov, Colored knot polynomials for arbitrary pretzel knots and links, Phys. Lett. B 743 (2015) 71 [arXiv: 1412.2616] [INSPIRE].

[61] D. Galakhov, D. Melnikov, A. Mironov and A. Morozov, Knot invariants from Virasoro related representation and pretzel knots, arXiv:1502.02621 [INSPIRE].

[62] M. Hara and M. Yamamoto, On Jones polynomials of alternating pretzel knots, J. Knot Theory Ramifications 21 (2012) 14 [arXiv:1112.2735].

[63] S. Nawata, P. Ramadevi and Zodinmawia, Colored HOMFLY polynomials from Chern-Simons theory, J. Knot Theor. 22 (2013) 1350078 [arXiv: 1302.5144] [INSPIRE].

[64] Knot Atlas at http://katlas.org/wiki/Main, D. Bar-Natan.

[65] I. Cherednik and I. Danilenko, DAHA and iterated torus knots, arXiv:1408.4348.

[66] R. Kashaev, The hyperbolic volume of knots from the quantum dilogarithm, Lett. Math. Phys. 39 (1997) 269 [q-alg/9601025].

[67] H. Murakami and J. Murakami, The colored Jones polynomials and the simplicial volume of a knot, Acta Math. 186 (2001) 85 [math/9905075].

[68] S. Gukov and H. Murakami, SL(2, C) Chern-Simons theory and the asymptotic behavior of the colored Jones polynomial, Lett. Math. Phys. 86 (2008) 79 [math/0608324] [INSPIRE]. 
[69] H. Murakami, An Introduction to the Volume Conjecture, arXiv:1002.0126.

[70] R. Gelca, On the relation between the A-polynomial and the Jones polynomia, Proc. Am. Math. Soc. 130 (2002) 1235 [math/0004158].

[71] R. Gelca and J. Sain, The noncommutative A-ideal of a $(2,2 p+1)$-torus knot determines its Jones polynomial, J. Knot Theory Ramifications 12 (2003) 187 [math/0201100].

[72] S. Gukov, Three-dimensional quantum gravity, Chern-Simons theory and the A polynomial, Commun. Math. Phys. 255 (2005) 577 [hep-th/0306165] [INSPIRE].

[73] S. Garoufalidis, On the characteristic and deformation varieties of a knot, Geom. Topol. Monogr. 7 (2004) 291 [math/0306230].

[74] M. Petkovsek, H.S. Wilf and D.Zeilberger, $A=B$, A.K. Peters Ltd., Wellesley, (1996).

[75] T.H. Koorwinder, On Zeilberger's algorithm and its q-analogue: a rigorous description, https://staff.fnwi.uva.nl/t.h.koornwinder/art/1993/zeilbalgo.pdf.

[76] D. Galakhov, A. Mironov and A. Morozov, Wall Crossing Invariants: from quantum mechanics to knots, J. Exp. Theor. Phys. 120 (2015) 549 [arXiv:1410.8482] [INSPIRE].

[77] L.D. Landau and E.M. Lifshitz, Quantum Mechanics: Non-Relativistic Theory, $3^{\text {rd }}$ edition, Pergamon Press, (1977).

[78] A.N. Kirillov and N.Yu. Reshetikhin, Representations of the Algebra $U_{q}(s l(2))$, q-Orthogonal Polynomials and Invariants of Links, Preprint, (1988).

[79] L. Álvarez-Gaumé, C. Gomez and G. Sierra, Quantum Group Interpretation of Some Conformal Field Theories, Phys. Lett. B 220 (1989) 142 [INSPIRE].

[80] S. Nawata, P. Ramadevi and Zodinmawia, Multiplicity-free quantum 6j-symbols for $U_{q}\left(s_{N}\right)$, Lett. Math. Phys. 103 (2013) 1389 [arXiv:1302.5143] [INSPIRE].

[81] R. Dijkgraaf, Mirror symmetry and elliptic curves, in The moduli spaces of curves, Birkhäuser, Prog. Math. 129 (1995) 149.

[82] P. Ramadevi, T.R. Govindarajan and R.K. Kaul, Chirality of knots 9(42) and 10(71) and Chern-Simons theory, Mod. Phys. Lett. A 9 (1994) 3205 [hep-th/9401095] [InSPIRE].

[83] A. Mironov, A. Morozov, A. Morozov, P. Ramadevi and V.K. Singh, Colored HOMFLY polynomials of knots presented as double fat diagrams, arXiv:1504.00371 [INSPIRE].

[84] S. Zhu, Colored HOMFLY polynomials via skein theory, JHEP 10 (2013) 229 [arXiv:1206.5886] [INSPIRE].

[85] M. Kontsevich, Vassiliev's Knot Invariants, Adv. Sov. Math. 16 (1993) 137.

[86] M. Alvarez, J.M.F. Labastida and E. Perez, Vassiliev invariants for links from Chern-Simons perturbation theory, Nucl. Phys. B 488 (1997) 677 [hep-th/9607030] [INSPIRE].

[87] P. Dunin-Barkowski, A. Sleptsov and A. Smirnov, Kontsevich Integral for Knots and Vassiliev Invariants, Int. J. Mod. Phys. A 28 (2013) 1330025 [arXiv:1112.5406] [InSPIRE].

[88] H. Morton and P. Cromwell, Distinguishing mutants by knot polynomials, J. Knot Theory Ramifications 5 (1996) 225.

[89] J. Murakami, Finite type invariants detecting the mutant knots, in Knot Theory: dedicated to Professor Kunio Murasugi for his 70th birthday, M. Sakuma et al. eds., Osaka University, (2000), pg. 258-267. 
[90] A. Morozov, Challenges of $\beta$-deformation, Theor. Math. Phys. 173 (2012) 1417 [arXiv:1201.4595] [INSPIRE].

[91] A. Mironov, A. Morozov, S. Shakirov and A. Sleptsov, Interplay between MacDonald and Hall-Littlewood expansions of extended torus superpolynomials, JHEP 05 (2012) 070 [arXiv:1201.3339] [INSPIRE].

[92] M. Khovanov and L. Rozansky, Matrix factorizations and link homology, Fund. Math. 199 (2008) 1 [math/0401268].

[93] M. Khovanov and L. Rozansky, Matrix factorizations and link homology II, Geom. Topol. 12 (2008) 1387 [math/0505056].

[94] M. Khovanov and L. Rozansky, Virtual crossings, convolutions and a categorification of the $\mathrm{SO}(2 N)$ Kauffman polynomial, math/0701333.

[95] V. Dolotin and A. Morozov, Introduction to Khovanov Homologies. III. A new and simple tensor-algebra construction of Khovanov-Rozansky invariants, Nucl. Phys. B 878 (2014) 12 [arXiv: 1308.5759] [INSPIRE].

[96] A. Anokhina and A. Morozov, Towards R-matrix construction of Khovanov-Rozansky polynomials. I. Primary T-deformation of HOMFLY, JHEP 07 (2014) 063 [arXiv: 1403.8087] [INSPIRE]. 